\title{
Synthesis and evaluation of analgesic, anti-inflammatory and ulcerogenic activities of some triazolo- and 2-pyrazolyl-pyrido[2,3- $d]$-pyrimidines
}

HEND N. HAFEZ

HEBAT-ALLAH S. ABBAS

ABDEL-RAHMAN B. A. EL-GAZZAR*

National Research Centre

Photochemistry Department

(Heterocyclic Unit), Dokki

12622 Cairo, Egypt

Accepted September 18, 2008
New series of 2-hydrazino-7,8-dihydro-6H-cyclopenta[5,6] pyrido[2,3-d]pyrimidines and its 1,7,8,9-tetrahydrocyclopenta[5,6]pyrido[2,3- $d][1,2,4]$ triazolo[ $[4,3-a]$ pyrimidine, 1,7 , 8,9-tetrahydrocyclopenta[5,6]pyrido[2,3- $d][1,2,3,4]$ tetrazolo[4,5-a]pyrimidine, 8,9-dihydro-7H-cyclopenta[5,6]pyrido[2,3-d]imidazolo[1,2-a]pyrimidine, 2-(pyrazol-1-yl)-7,8-dihydro-6H-cyclopenta[5,6]pyrido[2,3- $d$ ]pyrimidine derivatives were prepared in order to obtain new compounds with potential anti-inflammatory and analgesic activity and low ulcerogenic effect. The compounds possessing potent anti-inflammatory activity were further tested for their analgesic and ulcerogenic activities. Compounds 3-amino-6-(4-aryl)-9-(4-arylmethylene)-cyclopenta[5,6]pyrido[2,3 -d][1,2,4] triazolo[4,3-a]pyrimidin-5(H)-one (4c), 1-amino-2-methyl-6-(4-aryl)-9-(4-aryl-methylene)-cyclopenta[5,6]pyrido[2,3-d]imidazolo[1,2-a]pyrimidin-5(H)-one (6a), 2-amino-5-(4-aryl)-8-(4-arylmethylene)-cyclopenta[5,6]pyrido[2,3- $d$ ] pyrimidine-4(H)-one (9), 2-(3-amino-5-hydroxypyrazol-1-yl)-5-(4-aryl)-8-(4-arylmethylene)-cyclopenta[5,6] -pyrido[2,3- $d$ ]pyrimidin-4(H)-one (10a) and 3-thioxo-6-(4-aryl)-9-(4-arylmethylene)-cyclopenta[5,6]pyrido[2,3-d]$[1,2,4]$ triazolo[4,3-a]pyrimidin-5(H)-one (13) showed significant analgesic effects. Compound 2-(3-amino-5-hydroxypyrazol-1-yl)-5-(4-aryl)-8-(4-arylmethylene)-cyclopenta $[5,6]$ pyrido[2,3- $d]$ pyrimidin-4(H)-one (10a) was evaluated as the lead compound having higher anti-inflammatory activity $(82.8 \%)$ than ibuprofen $(79.5 \%)$ and lower ulcerogenic effect.

Keywords: pyrido[2,3-d]pyrimidines, [1,2,4]triazole anellation, anti-inflammatory, analgesic activity

It is already known that some pyrimido[4,5-b]quinolin-4-one derivatives display an interesting analgesic action in the writhing syndrome and hotplate tests and are not to-

\footnotetext{
* Correspondence, e-mail: profelgazzar@yahoo.com
} 
<smiles></smiles>

Fig. 1. Pyrido[2,3-d]pyrimidin-7(8H)-one

xic (1). Also, pyrido[2,3- $d]$ pyrimidin-7(8H)-ones have attracted interest of pharmaceutical companies due to the wide range of biological activities associated with this scaffold. Thus, a search revealed that more than 3000 structures of type A (Fig. 1) have been described; they show biological activities ranging from kinase inhibition (platelet-derived growth factor, PDGFr, fibroblast growth factor, FGFr, and epidermal growth factor, EGFr) (2), CSP/p38 kinase inhibition (3), Src tyrosine kinase inhibition (4, 5), cdk4 inhibition (6), p38 MAP kinase inhibition (7), cyclin dependent kinase inhibition $(8,9)$ telomerase inhibition (10) for the treatment of arthritis, Crohn's disease, irritable bowel syndrome, adult respiratory distress syndrome, chronic obstructive pulmonary disease, or Alzheimer's disease (11).

Moreover, due to their biological properties, which mainly depend on the nature and position of substituents, pyridopyrimidine derivatives are pharmaceutically active (12-17), including bactericidal (13), anticancer (14) and anti-inflammatory (17) activity. This prompted us to synthesize and identify new compounds derived from pyrido$[2,3-d]$ pyrimidin-4-ones and to screen them for analgesic and anti-inflammatory activities.

\section{EXPERIMENTAL}

All melting points were measured using an Electrothermal IA 9100 apparatus (Shimadzu, Japan) (Table I). ${ }^{1} \mathrm{H}$ NMR (Table II) and ${ }^{13} \mathrm{C}$ NMR spectra (Table III) were recorded on JEOL EX-270 and JEOL ECA-500 (Jeol, Japan) and chemical shifts were expressed as $\delta$ values against $\mathrm{Si}\left(\mathrm{CH}_{3}\right)_{4}$ as internal standard. IR spectra were recorded as $\mathrm{KBr}$ pellets on a Perkin-Elmer 1430 spectrometer (USA). Mass spectra (Table II) were recorded on a Hewlett-Packard-5988A GC/MS (USA) at ionization potential of $70 \mathrm{eV}$.

Synthesis of 5-aryl-8-arylmethylene-7,8-dihydro-2-thioxo-6H-cyclopenta[5,6]pyrido-[2,3-d]-pyrimidin-4(H)-ones $(\mathbf{1} a-c)$

The title compounds were prepared according to El-Gazzar et al. (18).

Synthesis of 5-aryl-8-arylmethylene-2-hydrazino-7,8-dihydro-6H-cyclopentena-[5,6]-pyrido$[2,3-\mathrm{d}]$ pyrimidin-4(H)-one $(2 a-c)$. General procedure

A suspension of compound 1a-c (0.01 mol) in hydrazine hydrate (99-100 \%) (25 mL) was stirred under reflux. The insoluble solid went into solution within 10 minutes with copious evolution of mercaptan to form a clear solution. After 30 minutes, heating was 
H. N. Hafez et al.: Synthesis and evaluation of analgesic, anti-inflammatory and ulcerogenic activities of some triazolo- and 2-pyrazolyl-pyrido[2,3-d]-pyrimidines, Acta Pharm. 58 (2008) 359-378.

continued for $8 \mathrm{~h}$ and the reaction mixture was allowed to cool to room temperature. The solid separated was filtered off, washed with ethanol and dried to produce $2 \mathrm{a}-\mathrm{c}$.

5-Phenyl-8-phenylmethylene-2-hydrazino-7,8-dihydro-6H-cyclopentena[5,6]pyrido-[2,3-d] pyrimidin-4(H)-one $(2 a)$. - Compound 2 a was obtained from $1 \mathbf{1 a}$ as a yellow powder which was crystallized from DMF.

5-(4-Chlorophenyl)-8-(4-chlorophenylmethylene)-2-hydrazino-7,8-dihydro-6H-cyclo-penta[5,6]pyrido[2,3-d]pyrimidin- $4(\mathrm{H})$-one $(\mathbf{2 b})$. - Compound $\mathbf{2 b}$ was obtained from $\mathbf{1 b}$ as a yellow powder which crystallized from dioxane.

5-(4-Methoxyphenyl)-8-(4-methoxyphenylmethylene)-2-hydrazino-7,8-dihydro-6H-cyclopenta[5,6]pyrido[2,3-d]pyrimidin-4(H)-one $(2 c)$. - Compound $2 \mathrm{c}$ was obtained from $1 \mathrm{c}$ as a yellow powder crystallized from ethanol/dioxane (1:1).

Synthesis of 5-(4-methoxyphenyl)-8-(4-methoxyphenylmethylene)-2-acethydrazido-7,8-dihydro-6H-cyclopenta[5,6]pyrido[2,3-d]pyrimidin-4(H)-one (3)

A solution of compound $2 \mathrm{c}(0.01 \mathrm{~mol})$ in glacial acetic acid, was refluxed for $3 \mathrm{~h}$. The reaction mixture was allowed to cool to room temperature and was poured into cold water $(100 \mathrm{~mL})$. The solid formed was collected by filtration, dried and crystallized from ethanol (dark yellow powder).

Synthesis of 6-(4-methoxyphenyl)-9-(4-methoxyphenylmethylene)-1,7,8,9-tetrahydrocyclopenta$[5,6]$ pyrido[2,3-d][1,2,4]triazolo[4,3-a]pyrimidin-5(H)-one $(4 a)$

A mixture of $2 \mathrm{c}(0.01 \mathrm{~mol})$ and formic acid $(10 \mathrm{~mL})$ and $2 \mathrm{~mL}$ of concentrated hydrochloric acid was heated under reflux for $8 \mathrm{~h}$. The reaction mixture was allowed to cool to room temperature and was poured into water $(100 \mathrm{~mL})$. The solid formed was collected by filtration, washed with ethanol $(20 \mathrm{~mL})$, dried and crystallized from DMF as an orange powder.

Synthesis of 6-(4-methoxyphenyl)-9-(4-methoxyphenylmethylene)-3-methyl-1,7,8,9-tetrahydrocyclopenta[5,6]pyrido[2,3-d][1,2,4]triazolo[4,3-a]pyrimidin-5(H)-one $(4 \boldsymbol{b})$

Method A. - A mixture of $2 \mathrm{c}(0.01 \mathrm{~mol})$ and glacial acetic acid $(50 \mathrm{~mL})$ was stirred under reflux for $10 \mathrm{~h}$ (TLC). The reaction mixture was allowed to cool to room temperature an was then poured into water $(100 \mathrm{~mL})$. The solid formed was collected by filtration, washed with ethanol $(20 \mathrm{~mL})$, dried, and crystallized from dioxane as an orange powder.

Method B. - A mixture of $3(0.01 \mathrm{~mol})$ and glacial acetic acid $(30 \mathrm{~mL})$ was stirred under reflux for $7 \mathrm{~h}$ (TLC). The reaction mixture was allowed to cool to room temperature and was then poured into water $(100 \mathrm{~mL})$. The solid formed was collected by filtration, washed with ethanol $(20 \mathrm{~mL})$ and crystallized.

Synthesis of 3-amino-6-(4-methoxyphenyl)-9-(4-methoxyphenylmethylene)-1,7,8,9-tetrahydrocyclopenta[5,6]pyrido[2,3-d][1,2,4]triazolo[4,3-a]pyrimidin-5(H)-one $(4 c)$

A mixture of $2 \mathrm{c}(0.01 \mathrm{~mol})$ and potassium thiocyanate $(0.15 \mathrm{~mol})$ was heated under reflux in glacial acetic acid $(30 \mathrm{~mL})$ for $6 \mathrm{~h}$. The reaction mixture was allowed to cool to room temperature and was poured into water. The precipitate formed was collected by filtration, dried and crystallized from ethanol/dioxane (2:1) as a yellow powder. 
H. N. Hafez et al.: Synthesis and evaluation of analgesic, anti-inflammatory and ulcerogenic activities of some triazolo- and 2-pyrazolyl-pyrido[2,3-d]-pyrimidines, Acta Pharm. 58 (2008) 359-378.

Synthesis of 3-aryl-6-(4-methoxyphenyl)-9-(4-methoxyphenylmethylene)-1,7,8,9-tetrahydrocyclopenta[5,6]pyrido[2,3-d][1,2,4]triazolo[4,3-a]pyrimidin-5(H)-one $(4 d-f)$. General procedure

A mixture of compound 7a-c $(0.01 \mathrm{~mol})$, anhydrous sodium acetate $(1.64 \mathrm{~g}, 0.02 \mathrm{~mol})$ and bromine $(1.60 \mathrm{~g}, 0.01 \mathrm{~mol})$ was stirred under reflux in glacial acetic acid $(30 \mathrm{~mL})$ in a water-bath at $80{ }^{\circ} \mathrm{C}$ for $20 \mathrm{~h}$ (under TLC control). The reaction mixture was allowed to cool to room temperature, was poured into water $(100 \mathrm{~mL})$ and the solid formed was collected by filtration and crystallized from appropriate solvent to afford $4 \mathbf{d}-\mathbf{f}$.

6-(4-Methoxyphenyl)-9-(4-methoxyphenylmethylene)-3-phenyl-1,7,8,9-tetrahydro-cyclopen ta[5,6]pyrido[2,3-d][1,2,4]triazolo[4,3-a]pyrimidin-5(H)-one $(4 d)$. - Compound $4 \mathrm{~d}$ was obtained from $7 \mathbf{a}$, as a yellow powder crystallized from dioxane.

3-(4-Chlorophenyl)-6-(4-methoxyphenyl)-9-(4-methoxyphenylmethylene)-1,7,8,9-tetrahydrocyclopenta[5,6]pyrido[2,3-d][1,2,4]triazolo[4,3-a]pyrimidin-5(H)-one (4e). - Compound 4e was obtained from $7 \mathbf{b}$ as a yellow powder crystallized from dioxane.

3,6-Di-(4-methoxyphenyl)-9-(4-methoxyphenylmethylene)-1,7,8,9-tetrahydrocyclo-penta[5,6] pyrido[2,3-d][1,2,4] triazolo[4,3-a]pyrimidin-5(H)-one $(4 f)$. - Compound $4 \mathrm{f}$ was obtained from $7 \mathrm{c}$ as a yellow powder which was crystallized from DMF.

Synthesis of 6-(4-methoxyphenyl)-9-(4-methoxyphenylmethylene)-8,9-dihydro-7H-cyclopenta-[5,6]pyrido[2,3-d][1,2,4]triazolo[4,3-a]pyrimidin-5(H)-one $(5 \boldsymbol{a}, \boldsymbol{b})$. General procedure

To a warmed ethanolic sodium ethoxide solution (prepared by dissolving $0.01 \mathrm{~mol}$ of sodium metal in $30 \mathrm{~mL}$ ethanol), each of $4 \mathbf{a}, \mathbf{b}(0.01 \mathrm{~mol})$, was added, the heating was continued for $30 \mathrm{~min}$ and the mixture was allowed to cool to room temperature. Then methyl iodide $(0.012 \mathrm{~mol})$ was added. The mixture was stirred under reflux for $3 \mathrm{~h}$, cooled to room temperature and poured into cold water $(100 \mathrm{~mL})$. The solid precipitated was filtered off, washed with water and dried to produce $5 \mathbf{a}, \mathbf{b}$.

1-Methyl-6-(4-methoxyphenyl)-9-(4-methoxyphenylmethylene)-8,9-dihydro-7H-cyclopenta$[5,6] p y r i d o[2,3-\mathrm{d}][1,2,4]$ triazolo[4,3-a]pyrimidin-5(H)-one $(5 a)$. - Compound 5a was obtained from $4 \mathbf{a}(10 \mathrm{mmol})$ and methyl iodide $(0.012 \mathrm{~mol})$ as yellow crystals crystallized from dioxane.

1,3-Dimethyl-6-(4-methoxyphenyl)-9-(4-methoxyphenylmethylene)-8,9-dihydro-7H-cyclopenta[5,6]pyrido[2,3-d][1,2,4]triazolo[4,3-a]pyrimidin-5(H)-one $(5 \boldsymbol{b})$. - Compound $5 \mathbf{b}$ was obtained from $4 \mathrm{~b}(10 \mathrm{mmol})$ and methyl iodide $(0.012 \mathrm{~mol})$ as a yellow powder, crystallized from dioxane.

Synthesis of 1-amino-2-methyl- or phenyl-6-(4-methoxyphenyl)-9-(4-methoxyphenyl-methylene)-8,9-dihydro-7H-cyclopenta[5,6]pyrido[2,3-d ]imidazolo[1,2-a]pyrimidin-5(H)-one $(6 \boldsymbol{a}, \boldsymbol{b})$.

General procedure

A mixture of compound $2 \mathrm{c}(0.01 \mathrm{~mol})$ and chloroacetone or 2-bromoacetophenone $(0.01 \mathrm{~mol})$ was heated under reflux for $12 \mathrm{~h}$ in dry xylene $(30 \mathrm{~mL})$. The solid that separated upon cooling was filtered off and crystallized from appropriate solvent to produce $6 a, b$.

1-Amino-2-methyl-6-(4-methoxyphenyl)-9-(4-methoxyphenylmethylene)-8,9-dihydro-7H-cyclopenta[5,6]pyrido[2,3-d] imidazolo[1,2-a]pyrimidin-5(H)-one $(6 a)$. - Compound 6 a was obtained from compound $2 \mathrm{c}(0.01 \mathrm{~mol})$ and chloroacetone $(0.01 \mathrm{~mol})$ as white crystals crystallized from ethanol. 
H. N. Hafez et al.: Synthesis and evaluation of analgesic, anti-inflammatory and ulcerogenic activities of some triazolo- and 2-pyrazolyl-pyrido[2,3-d]-pyrimidines, Acta Pharm. 58 (2008) 359-378.

1-Amino-2-phenyl-6-(4-methoxyphenyl)-9-(4-methoxyphenylmethylene)-8,9-dihydro-7H-cyclopenta[5,6]pyrido[2,3-d]imidazolo[1,2-a]pyrimidin-5(H)-one (6b). - Compound $6 \mathbf{b}$ was obtained from compound $2 \mathrm{c}(0.01 \mathrm{~mol})$ and 2-bromoacetophenone $(0.01 \mathrm{~mol})$ as a yellow powder crystallized from ethanol.

Synthesis of 2-arylmethylenehydrazone-5-(4-methoxyphenyl)-8-(4-methoxyphenylmethylene)-7,8-dihydro-6H-cyclopenta[5,6]pyrido[2,3-d]pyrimidin-4(H)-one (7a-c). General procedure

A mixture of $2 \mathrm{c}(0.01 \mathrm{~mol})$, the appropriate aromatic aldehyde $(0.01 \mathrm{~mol})$ and anhydrous sodium acetate $(0.02 \mathrm{~mol})$ was stirred under reflux in glacial acetic acid $(30 \mathrm{~mL})$ for $30 \mathrm{~min}$. The reaction mixture was allowed to cool to room temperature. The solid formed was filtered off and crystallized from appropriate solvent to produce $7 \mathbf{a}-\mathbf{c}$.

2-Phenylmethylenehydrazone-5-(4-methoxyphenyl)-8-(4-methoxyphenylmethylene)-7,8-dihydro-6H-cyclopenta[5,6]pyrido[2,3-d]pyrimidin-4(H)-one (7a). - Compound 7a was obtained from compound $2 \mathrm{c}(0.01 \mathrm{~mol})$ and benzaldehyde $(0.01 \mathrm{~mol})$ as pale yellow crystals crystallized from DMF.

2-(4-Chlorophenyl)methylenehydrazone-5-(4-methoxyphenyl)-8-(4-methoxyphenyl-methylene)7,8-dihydro-6H-cyclopenta[5,6]pyrido[2,3-d]pyrimidin-4(H)-one (7b). - Compound 7b was obtained from compound $2 \mathrm{c}(0.01 \mathrm{~mol})$ and 4 -chlorobenzaldehyde $(0.01 \mathrm{~mol})$ as pale yellow crystals crystallized from DMF.

2-(4-Methoxyphenyl)methylenehydrazone-5-(4-methoxyphenyl)-8-(4-methoxyphenyl-methylene)-7,8-dihydro-6H-cyclopenta[5,6]pyrido[2,3-d]pyrimidin-4(H)-one (7c). Compound 7c was obtained from compound $2 \mathrm{c}(0.01 \mathrm{~mol})$ and 4-methoxybenzaldehyde $(0.01 \mathrm{~mol})$ as a yellow powder which was crystallized from DMF.

Synthesis of 6-(4-methoxyphenyl)-9-(4-methoxyphenylmethylene)-1,7,8,9-tetrahydro-cyclopenta$[5,6]$ pyrido[2,3-d] $[1,2,3,4]$ tetrazolo[4,5-a]pyrimidin-5(H)-one $(8)$

To an ice-cold solution of compound $2 \mathrm{c}(0.01 \mathrm{~mol})$ in glacial acetic acid $(10 \mathrm{~mL})$, a solution of sodium nitrite $(0.15 \mathrm{~mol})$ in a small amount of water was added dropwise in an ice bath at $-5{ }^{\circ} \mathrm{C}$. The reaction mixture was allowed to stand overnight at room temperature and was then poured into water $(100 \mathrm{~mL})$. The solid precipitated was filtered off and crystallized from ethanol as a yellow powder.

Synthesis of 2-amino-5-(4-methoxyphenyl)-8-(4-methoxyphenylmethylene)-7,8-dihydro-6H-cyclopenta[5,6]pyrido[2,3-d]pyrimidin-4(H)-one (9)

To a well stirred solution of compound 8 (0.01 mol) in glacial acetic acid (30 mL), activated zinc dust $(5.00 \mathrm{~g})$ was added portion-wise at room temperature over a period of $30 \mathrm{~min}$. Stirring was continued for additional $3 \mathrm{~h}$. Thereafter, the reaction mixture was heated on a water bath $\left(80-90^{\circ} \mathrm{C}\right)$ for $3 \mathrm{~h}$. The reduction progress was monitored by TLC. After allowing the reaction mixture to cool to room temperature, it was poured into cold water $(100 \mathrm{~mL})$. The insoluble solid which separated was filtered, washed with water and dried. The crude solid was extracted with hot diethyl ether and the solid obtained after the removal of ether under reduced pressure was crystallized from ethanol. 
H. N. Hafez et al.: Synthesis and evaluation of analgesic, anti-inflammatory and ulcerogenic activities of some triazolo- and 2-pyrazolyl-pyrido[2,3-d]-pyrimidines, Acta Pharm. 58 (2008) 359-378.

Synthesis of 2-(3-amino-5-hydroxypyrazol-1-yl)-5-(4-methoxyphenyl)-8-(4-methoxyphenylmethylene)-7,8-dihydro-6H-cyclopenta[5,6]pyrido[2,3-d]pyrimidin-4(H)-one (10a)

To a warm ethanolic sodium ethoxide solution (prepared by dissolving $(0.01 \mathrm{~mol})$ sodium metal in absolute ethanol $(30 \mathrm{~mL})$, compound $2 \mathrm{c}(0.01 \mathrm{~mol})$ and ethyl cyanoacetate $(0.01 \mathrm{~mol})$ were added. The mixture was stirred under reflux for $8 \mathrm{~h}$, the reaction mixture was allowed to cool to room temperature was then poured into cold water (100 $\mathrm{mL}$ ) and neutralized with acetic acid. The solid product precipitated was filtered off, washed with water, ethanol, dried and crystallized from dioxane as a pale yellow powder.

Synthesis of 2-(sub-pyrazol-1-yl)-5-(4-methoxyphenyl)-8-(4-methoxyphenylmethylene)-7,8-dihydro$-6 \mathrm{H}-c y c l o p e n t a[5,6] p y r i d o[2,3-\mathrm{d}]$ pyrimidin-4(H)-one $(\mathbf{1 0 b}, \mathrm{c})$. General procedure

A mixture of compound $2 \mathrm{c}(0.01 \mathrm{~mol})$ and $\beta$-diketone $(0.01 \mathrm{~mol})$ in absolute ethanol $(30 \mathrm{~mL})$ was stirred under reflux for $5 \mathrm{~h}$. The reaction mixture was allowed to cool to 0 ${ }^{\circ} \mathrm{C}$ for $3 \mathrm{~h}$, the precipitate was filtered off, dried and crystallized from an appropriate solvent to produce $\mathbf{1 0 b}, \mathbf{c}$.

2-(3,5-Dimethyl-pyrazol-1-yl)-5-(4-methoxyphenyl)-8-(4-methoxyphenylmethylene)-7,8-dihydro$6 \mathrm{H}$-cyclopenta[5,6]pyrido[2,3-d]pyrimidin-4(H)-one (10b). - Compound $\mathbf{1 0 b}$ was obtained from $2 \mathrm{c}(0.01 \mathrm{~mol})$ with pentan-2,4-dione $(0.01 \mathrm{~mol})$ as pale light crystals crystallized from dioxane.

2-(3,5-Dimethyl-4-chloropyrazol-1-yl)-5-(4-methoxyphenyl)-8-(4-methoxyphenylmethylene)7,8-dihydro-6H-cyclopenta[5,6]pyrido[2,3-d]pyrimidin-4(H)-one (10c). - Compound 10c was obtained from $2 \mathrm{c}$ with 3 -chloropentan-2,4-dione $(0.01 \mathrm{~mol})$ as a light yellow powder crystallized from ethanol.

Synthesis of 2-[ethyl-5-(4-methoxyphenyl)-8-(4-methoxyphenylmethylene)-7,8-dihydro-6Hcyclopenta[5,6]pyrido[2,3-d]pyrimidin-4(H)-one acetate hydrazone (11)

A mixture of compound $2 \mathrm{c}(0.01 \mathrm{~mol})$ and ethyl acetoacetate $(0.01 \mathrm{~mol})$ was refluxed in absolute ethanol $(30 \mathrm{~mL})$ for $6 \mathrm{~h}$. The reaction mixture was allowed to cool to room temperature and the solid precipitate produced was filtered off and crystallized from ethanol as a pale brown powder.

Synthesis of 5-(4-methoxyphenyl)-8-(4-methoxyphenylmethylene)-2-(3-methyl-4H-pyrazol-5-one-1-yl)-7,8-dihydro-6H-cyclopenta[5,6]pyrido[2,3-d]pyrimidin-4(H)-one (12)

Method A. - A solution of compound $2 \mathrm{c}(0.01 \mathrm{~mol})$ and ethyl acetoacetate $(0.01 \mathrm{~mol})$ in sodium ethoxide solution (prepared by dissolving 0.01 mol of sodium metal in $30 \mathrm{~mL}$ absolute) ethanol was heated under reflux with stirring for $6 \mathrm{~h}$. The reaction mixture was allowed to cool and was poured into cold water $(100 \mathrm{~mL})$ and neutralized by acetic acid, whereby a solid was precipitated, filtered off and crystallized from ethanol to produce 12 as a yellow powder in $65 \%$ yield (m.p. $257-259{ }^{\circ} \mathrm{C}$, dec.).

Method B. - A solution of compound 11 (0.01 mol) was heated under reflux with sodium ethoxide solution (prepared by dissolving $0.01 \mathrm{~mol}$ of sodium metal in $30 \mathrm{~mL}$ absolute ethanol) for $3 \mathrm{~h}$. The reaction mixture was allowed to cool, was poured into water $(100 \mathrm{~mL})$ and neutralized by acetic acid; the precipitate formed was filtered off and crystallized from ethanol. 
H. N. Hafez et al.: Synthesis and evaluation of analgesic, anti-inflammatory and ulcerogenic activities of some triazolo- and 2-pyrazolyl-pyrido[2,3-d]-pyrimidines, Acta Pharm. 58 (2008) 359-378.

Synthesis of 3-thioxo-6-(4-methoxyphenyl)-9-(4-methoxyphenylmethylene)-1,2,8,9-tetrahydro-7H-cyclopenta[5,6]pyrido[2,3-d][1,2,4]triazolo[4,3-a]pyrimidin-5(H)-one (13)

To a warmed ethanolic sodium hydroxide solution prepared by dissolving $0.01 \mathrm{~mol}$ of sodium hydroxide in $50 \mathrm{~mL}$ ethanol, compound $2 \mathrm{c}(0.01 \mathrm{~mol})$ and excess carbon disulphide $(10 \mathrm{~mL})$ were added. The mixture was heated on a water-bath at $80{ }^{\circ} \mathrm{C}$ under reflux for $12 \mathrm{~h}$, was then allowed to cool to room temperature, poured onto water (100 $\mathrm{mL}$ ), neutralized by dilute acetic acid and the formed precipitate was filtered off and dried. The product was crystallized from ethanol as a yellow powder.

\section{Pharmacological screening}

Animals. - Adult male Sprague-Dawley rats, weighing 150-200 g, were used for anti-inflammatory and ulcerogenic activity testing, and Swiss albino mice of both sex, weighing 25-30 g, were used for analgesic activity testing. International principles and local regulations concerning the care and use of laboratory animals were observed (19). The animals had free access to standard commercial diet and water ad libitum and were kept in rooms maintained at $22 \pm 1^{\circ} \mathrm{C}$ with a $12 \mathrm{~h}$ light dark cycle. The experimental protocol was approved by the Animal Ethics Committee of the National Research Center, Cairo, Egypt.

Anti-inflammatory assay. - The compounds synthesized were evaluated for their anti-inflammatory activity using the carrageenean induced hind paw edema method (20). The animals were randomly allocated to groups of six animals each and were fasted for $24 \mathrm{~h}$ before the experiment, with free access to water. Control group received only $0.5 \%(\mathrm{~m} / \mathrm{V})$ carboxymethyl cellulose solution. Standard drug ibuprofen was administrated orally at a 30 $\mathrm{mg} \mathrm{kg}^{-1}$ dose. Carrageenean solution in saline $(1 \%, 0.1 \mathrm{~mL})$ was injected subcutaneously into the sub-plantar region of the left hind paw of each rat, one hour after the administration of the test compounds or standard drug $\left(30 \mathrm{mg} \mathrm{kg}^{-1}\right)$. The left hind paw volume was measured before and after 3 and $4 \mathrm{~h}$ of carrageenean treatment by means of a plethysmometer. The percent edema inhibition was calculated from the mean effect in the control and treated animals. Each value represents the mean \pm SEM relative to the standard and data were analyzed by ANOVA followed by Dunnett's test.

Analgesic assay. - Analgesic activity was evaluated by the tail immersion method (21). Swiss albino mice allocated to different groups consisting of six animals each were used for the experiment. Analgesic activity was evaluated after oral administration of the test compounds at a dose of $30 \mathrm{mg} \mathrm{kg}^{-1}$ of ibuprofen and the test compounds. Test compounds and the standard drug were administered orally as a suspension in carboxylmethyl cellulose solution in water $(0.5 \%, \mathrm{~m} / \mathrm{V})$. The analgesic activity was assessed before and after $4 \mathrm{~h}$ following administration of test compounds and standard drug. The lower 5 -cm portion of the tail was gently immersed into thermostatically controlled water at $55 \pm 0.5{ }^{\circ} \mathrm{C}$. The time in seconds for tail withdrawal from water was taken to be the reaction time with a cut off time of immersion, set at 100 seconds for both control as well as treated groups of animals.

Ulcerogenicity. - Acute ulcerogenicity was determined according to the method of Cioli et al. (22). The animals were allocated to different groups consisting of six animals each. Ulcerogenic activity was evaluated after oral administration of the test compounds 
at a dose of $30 \mathrm{mg} \mathrm{kg}^{-1}$. Control group received only $0.5 \%(\mathrm{~m} / \mathrm{V})$ carboxymethyl cellulose solution. After the drug treatment, the rats were fed normal diet for $17 \mathrm{~h}$ and were then sacrificed. The stomach was removed and opened along the greater curvature, washed with distilled water and cleaned gently by dipping in normal saline. The mucosal damage was examined by means of a magnifying glass. Mucosal damage was assessed for each stomach according to the following scoring system: 0.5: redness, 1.0: spot ulcers, 1.5: hemorrhagic streaks, 2.0: $3<$ ulcers $\leq 5,3.0$ : ulcers $>5$. The mean score of each treated group minus the mean score of the control group was regarded as the severity index of gastric mucosal damage.

\section{RESULTS AND DISCUSSION}

5-Aryl-8-arylmethylene-7,8-dihydro-2-thioxo-6H-cyclopenta[5,6]pyrido[2,3-d]-pyrimidin-4(H)-ones (1a-c) were synthesized previously (18). Beside the correct values in elemental analyses and spectral data, structures were established chemically. Upon treatment with hydrazine hydrate (Scheme 1), thay gave 5-aryl-8-arylmethylene-2-hydrazino-7,8-dihydro-6H-cyclopenta[5,6]pyrido-[2,3-d] pyrimidin-4(H)-ones (2a-c), with the evolution of mercaptan.

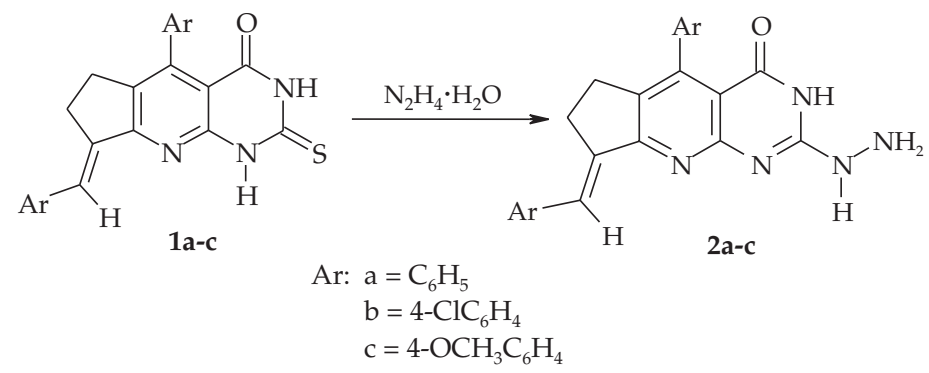

Scheme 1

Compound $2 \mathrm{c}$ could be considered as a starting material for the synthesis of new polynuclear heterocycles such as azolopyridopyrimidines, as well as the synthesis of some pyrazolopyridopyrimidine derivatives. Thus, heating compound $2 \mathrm{c}$ with aliphatic acids, namely, formic and acetic acid, resulted in the formation of 6-(4-methoxyphenyl)-9-(4-methoxyphenylmethylene)-1,7,8,9-tetrahydro-cyclopenta[5,6]pyrido[2,3- $d][1,2,4]$ triazolo-[4,3-a]pyrimidin-5(H)-ones $(4 \mathbf{a}, \mathbf{b})$. On the other hand, heating of compound $2 \mathbf{c}$ with acetic acid for 3 hours only yielded 2-acetylhydrazino derivative 3 , which on further long heating with acetic acid gave $4 \mathbf{b}$. Moreover, alkylation of $4 \mathbf{a}, \mathbf{b}$ in ethanolic sodium ethoxide solution with methyl iodide afforded 1-methyl-6-(4-methoxyphenyl)-9-(4-methoxyphenylmethylene)-8,9-dihydrocyclopenta[5,6]pyrido[2,3- $d][1,2,4]$ triazolo[4,3-a ] pyrimidin-5(H)-ones $(\mathbf{5} \mathbf{a}, \mathbf{b})$ as shown in Scheme 2 . All the synthesized compounds were characterized by their physical, chemical and spectral data (Tables I-III). IR spectra of compounds $4 \mathbf{a}, \mathbf{b}$ showed the presence of characteristic absorption peaks around 3385-3400 
H. N. Hafez et al.: Synthesis and evaluation of analgesic, anti-inflammatory and ulcerogenic activities of some triazolo- and 2-pyrazolyl-pyrido[2,3-d]-pyrimidines, Acta Pharm. 58 (2008) 359-378.

Table I. Physical and chemical properties of synthesized compounds

\begin{tabular}{|c|c|c|c|c|c|c|}
\hline \multirow{2}{*}{$\begin{array}{l}\text { Compd. } \\
\text { No. }\end{array}$} & \multirow{2}{*}{$\begin{array}{l}\text { Yield } \\
(\%)\end{array}$} & \multirow{2}{*}{$\begin{array}{l}\text { M.p. } \\
\left({ }^{\circ} \mathrm{C}\right)\end{array}$} & \multirow{2}{*}{$\begin{array}{l}\text { Mol. formula } \\
\qquad\left(M_{\mathrm{r}}\right)\end{array}$} & \multicolumn{3}{|c|}{ Found/calcd. (\%) } \\
\hline & & & & $\mathrm{N}$ & $\mathrm{H}$ & $\mathrm{C}$ \\
\hline $2 a$ & 86 & $248-250$ & $\begin{array}{c}\mathrm{C}_{23} \mathrm{H}_{19} \mathrm{~N}_{5} \mathrm{O} \\
(381.4)\end{array}$ & $\begin{array}{l}72.42 \\
72.37\end{array}$ & $\begin{array}{l}5.02 \\
5.00\end{array}$ & $\begin{array}{l}18.36 \\
18.29\end{array}$ \\
\hline $2 b$ & 89 & $233-236$ & $\begin{array}{c}\mathrm{C}_{23} \mathrm{H}_{17} \mathrm{Cl}_{2} \mathrm{~N}_{5} \mathrm{O} \\
(450.3)\end{array}$ & $\begin{array}{l}61.34 \\
61.29\end{array}$ & $\begin{array}{l}3.81 \\
3.79\end{array}$ & $\begin{array}{l}15.55 \\
15.58\end{array}$ \\
\hline $2 c$ & 87 & $282-284$ & $\begin{array}{c}\mathrm{C}_{25} \mathrm{H}_{23} \mathrm{~N}_{5} \mathrm{O}_{3} \\
(441.5)\end{array}$ & $\begin{array}{l}68.01 \\
68.03\end{array}$ & $\begin{array}{l}5.25 \\
5.28\end{array}$ & $\begin{array}{l}15.86 \\
15.79\end{array}$ \\
\hline 3 & 75 & $267-270$ & $\begin{array}{c}\mathrm{C}_{27} \mathrm{H}_{25} \mathrm{~N}_{5} \mathrm{O}_{4} \\
(483.5)\end{array}$ & $\begin{array}{l}67.06 \\
67.04\end{array}$ & $\begin{array}{l}5.21 \\
5.18\end{array}$ & $\begin{array}{l}14.48 \\
14.51\end{array}$ \\
\hline $4 a$ & 67 & $309-311$ & $\begin{array}{c}\mathrm{C}_{26} \mathrm{H}_{21} \mathrm{~N}_{5} \mathrm{O}_{3} \\
(451.5)\end{array}$ & $\begin{array}{l}69.16 \\
69.13\end{array}$ & $\begin{array}{l}4.69 \\
4.71\end{array}$ & $\begin{array}{l}15.51 \\
15.48\end{array}$ \\
\hline $4 b$ & 64 & $339-341$ & $\begin{array}{c}\mathrm{C}_{27} \mathrm{H}_{23} \mathrm{~N}_{5} \mathrm{O}_{3} \\
(465.5)\end{array}$ & $\begin{array}{l}69.66 \\
69.63\end{array}$ & $\begin{array}{l}4.98 \\
4.96\end{array}$ & $\begin{array}{l}15.04 \\
15.07\end{array}$ \\
\hline $4 c$ & 73 & $336-338$ & $\begin{array}{c}\mathrm{C}_{26} \mathrm{H}_{22} \mathrm{~N}_{6} \mathrm{O}_{3} \\
(466.5)\end{array}$ & $\begin{array}{l}66.94 \\
66.91\end{array}$ & $\begin{array}{l}4.75 \\
4.69\end{array}$ & $\begin{array}{l}18.02 \\
18.04\end{array}$ \\
\hline $4 d$ & 60 & $320-322$ & $\begin{array}{c}\mathrm{C}_{32} \mathrm{H}_{25} \mathrm{~N}_{5} \mathrm{O}_{3} \\
(527.5)\end{array}$ & $\begin{array}{l}72.85 \\
72.83\end{array}$ & $\begin{array}{l}4.78 \\
4.75\end{array}$ & $\begin{array}{l}13.27 \\
13.25\end{array}$ \\
\hline $4 e$ & 63 & $300-302$ & $\begin{array}{c}\mathrm{C}_{32} \mathrm{H}_{24} \mathrm{ClN}_{5} \mathrm{O}_{3} \\
(562.1)\end{array}$ & $\begin{array}{l}68.38 \\
68.35\end{array}$ & $\begin{array}{l}4.30 \\
4.27\end{array}$ & $\begin{array}{l}12.46 \\
12.43\end{array}$ \\
\hline $4 f$ & 64 & $343-345$ & $\begin{array}{c}\mathrm{C}_{33} \mathrm{H}_{27} \mathrm{~N}_{5} \mathrm{O}_{4} \\
(557.6)\end{array}$ & $\begin{array}{l}71.08 \\
71.05\end{array}$ & $\begin{array}{l}4.88 \\
4.87\end{array}$ & $\begin{array}{l}12.56 \\
12.59\end{array}$ \\
\hline $5 a$ & 68 & $278-280$ & $\begin{array}{c}\mathrm{C}_{27} \mathrm{H}_{23} \mathrm{~N}_{5} \mathrm{O}_{3} \\
(465.5)\end{array}$ & $\begin{array}{l}69.66 \\
69.62\end{array}$ & $\begin{array}{l}4.97 \\
4.98\end{array}$ & $\begin{array}{l}15.04 \\
15.01\end{array}$ \\
\hline $5 b$ & 70 & $263-265$ & $\begin{array}{c}\mathrm{C}_{28} \mathrm{H}_{25} \mathrm{~N}_{5} \mathrm{O}_{3} \\
(479.5)\end{array}$ & $\begin{array}{l}70.13 \\
69.98\end{array}$ & $\begin{array}{l}5.25 \\
5.20\end{array}$ & $\begin{array}{l}14.60 \\
14.58\end{array}$ \\
\hline $6 a$ & 68 & $264-266$ & $\begin{array}{c}\mathrm{C}_{28} \mathrm{H}_{25} \mathrm{~N}_{5} \mathrm{O}_{3} \\
(479.5)\end{array}$ & $\begin{array}{l}70.13 \\
70.09\end{array}$ & $\begin{array}{l}5.26 \\
5.19\end{array}$ & $\begin{array}{l}14.60 \\
14.63\end{array}$ \\
\hline $6 b$ & 65 & $248-250$ & $\begin{array}{c}\mathrm{C}_{33} \mathrm{H}_{27} \mathrm{~N}_{5} \mathrm{O}_{3} \\
(541.6)\end{array}$ & $\begin{array}{l}73.17 \\
73.19\end{array}$ & $\begin{array}{l}5.02 \\
4.99\end{array}$ & $\begin{array}{l}12.93 \\
12.94\end{array}$ \\
\hline $7 a$ & 89 & $295-297$ & $\begin{array}{c}\mathrm{C}_{32} \mathrm{H}_{27} \mathrm{~N}_{5} \mathrm{O}_{3} \\
(529.6)\end{array}$ & $\begin{array}{l}72.57 \\
72.54\end{array}$ & $\begin{array}{l}5.14 \\
5.09\end{array}$ & $\begin{array}{l}13.22 \\
13.18\end{array}$ \\
\hline $7 \mathrm{~b}$ & 83 & $288-290$ & $\begin{array}{c}\mathrm{C}_{32} \mathrm{H}_{26} \mathrm{ClN}_{5} \mathrm{O}_{3} \\
(564.1)\end{array}$ & $\begin{array}{l}68.14 \\
68.09\end{array}$ & $\begin{array}{l}4.65 \\
4.62\end{array}$ & $\begin{array}{l}12.41 \\
12.39\end{array}$ \\
\hline $7 c$ & 81 & $330-332$ & $\begin{array}{c}\mathrm{C}_{33} \mathrm{H}_{29} \mathrm{~N}_{5} \mathrm{O}_{4} \\
(559.6)\end{array}$ & $\begin{array}{l}70.83 \\
70.79\end{array}$ & $\begin{array}{l}5.22 \\
5.23\end{array}$ & $\begin{array}{l}12.51 \\
12.48\end{array}$ \\
\hline 8 & 53 & $260-262$ & $\begin{array}{c}\mathrm{C}_{25} \mathrm{H}_{20} \mathrm{~N}_{6} \mathrm{O}_{3} \\
(452.5)\end{array}$ & $\begin{array}{l}66.36 \\
66.34\end{array}$ & $\begin{array}{l}4.46 \\
4.47\end{array}$ & $\begin{array}{l}18.57 \\
18.59\end{array}$ \\
\hline 9 & 49 & $280-282$ & $\begin{array}{c}\mathrm{C}_{25} \mathrm{H}_{22} \mathrm{~N}_{4} \mathrm{O}_{3} \\
(426.4)\end{array}$ & $\begin{array}{l}70.40 \\
70.38\end{array}$ & $\begin{array}{l}5.20 \\
5.17\end{array}$ & $\begin{array}{l}13.14 \\
13.09\end{array}$ \\
\hline $10 a$ & 73 & $258-260$ & $\begin{array}{c}\mathrm{C}_{28} \mathrm{H}_{24} \mathrm{~N}_{6} \mathrm{O}_{4} \\
(508.5)\end{array}$ & $\begin{array}{l}66.13 \\
66.09\end{array}$ & $\begin{array}{l}4.76 \\
4.74\end{array}$ & $\begin{array}{l}16.53 \\
16.49\end{array}$ \\
\hline
\end{tabular}


H. N. Hafez et al.: Synthesis and evaluation of analgesic, anti-inflammatory and ulcerogenic activities of some triazolo- and 2-pyrazolyl-pyrido[2,3-d]-pyrimidines, Acta Pharm. 58 (2008) 359-378.

\begin{tabular}{|c|c|c|c|c|c|c|}
\hline $10 \mathrm{~b}$ & 78 & $240-242$ & $\begin{array}{c}\mathrm{C}_{30} \mathrm{H}_{27} \mathrm{~N}_{5} \mathrm{O}_{3} \\
(505.5)\end{array}$ & $\begin{array}{l}71.27 \\
71.25\end{array}$ & $\begin{array}{l}5.38 \\
5.36\end{array}$ & $\begin{array}{l}13.85 \\
13.79\end{array}$ \\
\hline $10 c$ & 77 & $266-268$ & $\begin{array}{c}\mathrm{C}_{30} \mathrm{H}_{26} \mathrm{ClN}_{5} \mathrm{O}_{3} \\
(540.0)\end{array}$ & $\begin{array}{l}66.72 \\
66.68\end{array}$ & $\begin{array}{l}4.85 \\
4.82\end{array}$ & $\begin{array}{l}12.97 \\
12.95\end{array}$ \\
\hline 11 & 68 & $235-237$ & $\begin{array}{c}\mathrm{C}_{31} \mathrm{H}_{31} \mathrm{~N}_{5} \mathrm{O}_{5} \\
(553.6)\end{array}$ & $\begin{array}{l}67.25 \\
67.23\end{array}$ & $\begin{array}{l}5.64 \\
5.66\end{array}$ & $\begin{array}{l}12.65 \\
12.63\end{array}$ \\
\hline 12 & 65 & $257-259$ & $\begin{array}{c}\mathrm{C}_{29} \mathrm{H}_{25} \mathrm{~N}_{5} \mathrm{O}_{4} \\
(507.5)\end{array}$ & $\begin{array}{l}68.62 \\
68.59\end{array}$ & $\begin{array}{l}4.96 \\
4.99\end{array}$ & $\begin{array}{l}13.80 \\
13.75\end{array}$ \\
\hline 13 & 76 & $311-313$ & $\begin{array}{c}\mathrm{C}_{26} \mathrm{H}_{21} \mathrm{~N}_{5} \mathrm{O}_{3} \mathrm{~S} \\
(483.5)\end{array}$ & $\begin{array}{l}64.58 \\
64.59\end{array}$ & $\begin{array}{l}4.38 \\
4.35\end{array}$ & $\begin{array}{l}14.48 \\
14.39\end{array}$ \\
\hline
\end{tabular}

Table II. Spectral data of synthesized compounds

\begin{tabular}{|c|c|c|c|}
\hline $\begin{array}{l}\text { Compd. } \\
\text { No. }\end{array}$ & $\begin{array}{l}\text { Mass } \\
(\mathrm{m} / \mathrm{z})\end{array}$ & $\begin{array}{c}\mathrm{IR} \\
\left(v, \mathrm{~cm}^{-1}\right)\end{array}$ & $\begin{array}{c}{ }^{1} \mathrm{H} \text { NMR }(\delta, \mathrm{ppm}) \\
\left(\mathrm{DMSO}-d_{6}\right)\end{array}$ \\
\hline $2 a$ & $\begin{array}{l}\text { MS }(m / z) \\
381\left(\mathrm{M}^{+}\right)\end{array}$ & $\begin{array}{l}3400(\mathrm{br}, \mathrm{NH}) \\
1687(\mathrm{CO}) \\
1625(\mathrm{C}=\mathrm{N})\end{array}$ & $\begin{array}{l}2.05\left(\mathrm{br}, 2 \mathrm{H}, \mathrm{NH}_{2}\right), 2.71-2.75\left(\mathrm{~m}, 2 \mathrm{H}, \mathrm{CH}_{2}\right), 2.84-2.95(\mathrm{t}, \\
\left.2 \mathrm{H}, \mathrm{CH}_{2}\right), 5.15(\mathrm{br}, \mathrm{NH}), 7.07-7.11(\mathrm{~m}, 2 \mathrm{H}, \mathrm{Ar}-\mathrm{H}), \\
7.32-7.39(\mathrm{~m}, 3 \mathrm{H}, \mathrm{Ar}-\mathrm{H}), 7.45-7.59(\mathrm{~m}, 5 \mathrm{H}, \mathrm{Ar}-\mathrm{H}) \text { and } \\
8.22(\mathrm{~s}, 1 \mathrm{H}, \mathrm{CH}), 9.10(\mathrm{br}, \mathrm{NH})\end{array}$ \\
\hline
\end{tabular}

2b $\quad$ MS (m/z), 3410 (brs, NH), $2.10\left(\mathrm{br}, 2 \mathrm{H}, \mathrm{NH}_{2}\right), 2.73-2.76\left(\mathrm{~m}, 2 \mathrm{H}, \mathrm{CH}_{2}\right), 2.80-2.92(\mathrm{~m}$, $\left.452\left(\mathrm{M}^{+}+2\right), \quad 1686(\mathrm{CO}), \quad 2 \mathrm{H}, \mathrm{CH}_{2}\right), 3.80(\mathrm{br}, \mathrm{NH}), 6.98-7.05(\mathrm{~d}, 2 \mathrm{H}, J=8.43 \mathrm{~Hz}$, $\left.450\left(\mathrm{M}^{+}\right) \quad 1640(\mathrm{C}=\mathrm{N}) \quad \operatorname{Ar}-\mathrm{H}\right), 7.10-7.19(\mathrm{~d}, 2 \mathrm{H}, J=8,42 \mathrm{~Hz}, \mathrm{Ar}-\mathrm{H}), 7.43-7.50(\mathrm{~d}$, $2 \mathrm{H}, J=8.40 \mathrm{~Hz}, \mathrm{Ar}-\mathrm{H}), 7.54-7.60(\mathrm{~d}, 2 \mathrm{H}, J=8.40 \mathrm{~Hz}$, Ar-H), $8.02(\mathrm{~s}, 1 \mathrm{H}, \mathrm{CH}), 12.00(\mathrm{br}, \mathrm{NH})\left(2 \mathrm{NH}, \mathrm{NH}_{2}, \mathrm{D}_{2} \mathrm{O}\right.$ exchangeable)

2c $\quad$ MS (m/z), $3395(\mathrm{br}, \mathrm{NH}), 2.00\left(\mathrm{br}, 2 \mathrm{H}, \mathrm{NH}_{2}\right), 2.70-2.72\left(\mathrm{~m}, 2 \mathrm{H}, \mathrm{CH}_{2}\right), 2.80-2.90(\mathrm{~m}$, $\left.441\left(\mathrm{M}^{+}\right) \quad 1689(\mathrm{CO}), \quad 2 \mathrm{H}, \mathrm{CH}_{2}\right), 3.80,3.83\left(2 \mathrm{~s}, 6 \mathrm{H}, 2 \mathrm{OCH}_{3}\right), 5.15(\mathrm{br}, \mathrm{NH})$, $1646(\mathrm{C}=\mathrm{N}) \quad 6.92-6.98(\mathrm{~d}, 2 \mathrm{H}, J=8.41 \mathrm{~Hz}, \mathrm{Ar}-\mathrm{H}), 7.00-7.19(\mathrm{~d}, 2 \mathrm{H}, J$ $=8.41 \mathrm{~Hz}, \mathrm{Ar}-\mathrm{H}), 7.50-7.53(\mathrm{~d}, 2 \mathrm{H}, J=8.39 \mathrm{~Hz}, \mathrm{Ar}-\mathrm{H})$, 7.56-7.61 (d, 2H, $J=8.40 \mathrm{~Hz}, \mathrm{Ar}-\mathrm{H}), 8.16(\mathrm{~s}, 1 \mathrm{H}, \mathrm{CH})$, 11.50 (br, NH) $\left(2 \mathrm{NH}, \mathrm{NH}_{2}, \mathrm{D}_{2} \mathrm{O}\right.$ exchangeable)

$3 \quad \mathrm{MS}(\mathrm{m} / \mathrm{z}), \quad 3400(\mathrm{br}, \mathrm{NH}), \quad 2.73-2.78\left(\mathrm{~m}, 2 \mathrm{H}, \mathrm{CH}_{2}\right), 2.82-2.91\left(\mathrm{~m}, 2 \mathrm{H}, \mathrm{CH}_{2}\right), 2.86(\mathrm{~s}$, $\left.468 \quad 1688,1680 \quad 3 \mathrm{H}, \mathrm{CH}_{3}\right), 3.82,3.86\left(2 \mathrm{~s}, 6 \mathrm{H}, 2 \mathrm{OCH}_{3}\right), 6.96(\mathrm{~d}, 2 \mathrm{H}, J=$ $\left.\left(\mathrm{M}^{+}-\mathrm{CH}_{3}\right) \quad(2 \mathrm{CO}), \quad 8.39 \mathrm{~Hz}, \mathrm{Ar}-\mathrm{H}\right), 7.11(\mathrm{~d}, 2 \mathrm{H}, J=8.40 \mathrm{~Hz}, \mathrm{Ar}-\mathrm{H}), 7.49(\mathrm{~d}$, $1620(\mathrm{C}=\mathrm{N}) \quad 2 \mathrm{H}, J=8.39 \mathrm{~Hz}, \mathrm{Ar}-\mathrm{H}), 7.60(\mathrm{~d}, 2 \mathrm{H}, J=8.40 \mathrm{~Hz}, \mathrm{Ar}-\mathrm{H})$, $8.19(\mathrm{~s}, 1 \mathrm{H}, \mathrm{CH}), 8.90,9.30,11.10\left(3 \mathrm{br}, 3 \mathrm{NH}, \mathrm{D}_{2} \mathrm{O}\right.$ exchangeable)

4a $\quad$ MS $(\mathrm{m} / \mathrm{z}), \quad 3385(\mathrm{br}, \mathrm{NH}), \quad 2.72-2.76\left(\mathrm{~m}, 2 \mathrm{H}, \mathrm{CH}_{2}\right), 2.85-2.91\left(\mathrm{~m}, 2 \mathrm{H}, \mathrm{CH}_{2}\right), 3.83$, $451\left(\mathrm{M}^{+}\right) \quad 1687(\mathrm{CO}), \quad 3.89\left(2 \mathrm{~s}, 6 \mathrm{H}, 2 \mathrm{OCH}_{3}\right), 6.98-7.04(\mathrm{~d}, 2 \mathrm{H}, \mathrm{Ar}-\mathrm{H}), 7.08-7.15$ $1615(\mathrm{C}=\mathrm{N}) \quad(\mathrm{d}, 2 \mathrm{H}, \mathrm{Ar}-\mathrm{H}), 7.45-7.50(\mathrm{~d}, 2 \mathrm{H}, \mathrm{Ar}-\mathrm{H}), 7.56-7.63(\mathrm{~d}, 2 \mathrm{H}$, Ar- $\mathrm{H}), 8.12(\mathrm{~s}, 1 \mathrm{H}, \mathrm{CH}), 8.54(\mathrm{~s}, 1 \mathrm{H}$, triazole proton) 9.50 (br, $\mathrm{NH}, \mathrm{D}_{2} \mathrm{O}$ exchangeable)

4b $\quad$ MS $(\mathrm{m} / \mathrm{z}), \quad 3400(\mathrm{br}, \mathrm{NH}), \quad 2.25\left(\mathrm{~s}, 3 \mathrm{H}, \mathrm{CH}_{3}\right), 2.71-2.77\left(\mathrm{~m}, 2 \mathrm{H}, \mathrm{CH}_{2}\right), 2.85-2.91(\mathrm{~m}$, $\left.465\left(\mathrm{M}^{+}\right) \quad 1685(\mathrm{CO}), \quad 2 \mathrm{H}, \mathrm{CH}_{2}\right), 3.86,3.91\left(2 \mathrm{~s}, 6 \mathrm{H}, 2 \mathrm{OCH}_{3}\right), 7.04(\mathrm{~d}, 2 \mathrm{H}, J=$ $1620(\mathrm{C}=\mathrm{N}) \quad 8.39 \mathrm{~Hz}, \mathrm{Ar}-\mathrm{H}), 7.15(\mathrm{~d}, 2 \mathrm{H}, \mathrm{J}=8.38 \mathrm{~Hz}, \mathrm{Ar}-\mathrm{H}), 7.47(\mathrm{~d}$, $2 \mathrm{H}, J=8.38 \mathrm{~Hz}, \mathrm{Ar}-\mathrm{H}), 7.59(\mathrm{~d}, 2 \mathrm{H}, J=8.38 \mathrm{~Hz}, \mathrm{Ar}-\mathrm{H})$, $8.08(\mathrm{~s}, 1 \mathrm{H}, \mathrm{CH}), 10.20$ (br, $\mathrm{NH}, \mathrm{D}_{2} \mathrm{O}$ exchangeable) 
H. N. Hafez et al.: Synthesis and evaluation of analgesic, anti-inflammatory and ulcerogenic activities of some triazolo- and 2-pyrazolyl-pyrido[2,3-d]-pyrimidines, Acta Pharm. 58 (2008) 359-378.

$4 c$

MS $(m / z)$, $466\left(\mathrm{M}^{+}\right)$

4d

$4 e$

$4 f$

$5 a$

$5 b$

$6 a$

$6 \mathrm{~b}$

$7 a$
3420 (br, NH), $2.74-2.78\left(\mathrm{~m}, 2 \mathrm{H}, \mathrm{CH}_{2}\right), 2.84-2.90\left(\mathrm{~m}, 2 \mathrm{H}, \mathrm{CH}_{2}\right), 3.85$, $1686(\mathrm{CO}), \quad 3.88\left(2 \mathrm{~s}, 6 \mathrm{H}, 2 \mathrm{OCH}_{3}\right), 5.54\left(\mathrm{br}, \mathrm{NH}_{2}\right), 6.99(\mathrm{~d}, 2 \mathrm{H}, \mathrm{J}=$ $1646(\mathrm{C}=\mathrm{N}) \quad 8.38 \mathrm{~Hz}, \mathrm{Ar}-\mathrm{H}), 7.10(\mathrm{~d}, 2 \mathrm{H}, J=8.39 \mathrm{~Hz}, \mathrm{Ar}-\mathrm{H}), 7.49$ (d, $2 \mathrm{H}, J=8.40 \mathrm{~Hz}, \mathrm{Ar}-\mathrm{H}), 7.61(\mathrm{~d}, 2 \mathrm{H}, J=8.40 \mathrm{~Hz}, \mathrm{Ar}-\mathrm{H})$, 8.22 (s, 1H, CH), 10.50 (br, NH) (NH, $\mathrm{NH}_{2} \mathrm{D}_{2} \mathrm{O}$ exchangeable)

3410 (br, NH), $2.73-2.82\left(\mathrm{~m}, 2 \mathrm{H}, \mathrm{CH}_{2}\right), 2.84-2.92\left(\mathrm{~m}, 2 \mathrm{H}, \mathrm{CH}_{2}\right), 3.85$, $1698(\mathrm{CO}), \quad 3.90\left(2 \mathrm{~s}, 6 \mathrm{H}, 2 \mathrm{OCH}_{3}\right), 6.95-6.99(\mathrm{~d}, 2 \mathrm{H}, \mathrm{Ar}-\mathrm{H}), 7.03-7.15$ $1615(\mathrm{C}=\mathrm{N}) \quad(\mathrm{m}, 2 \mathrm{H}, \mathrm{Ar}-\mathrm{H}), 7.18-7.21(\mathrm{~d}, 2 \mathrm{H}, \mathrm{Ar}-\mathrm{H}), 7.33-7.43(\mathrm{~m}$, $3 \mathrm{H}, \mathrm{Ar}-\mathrm{H}), 7.54-7.58$ (d, 2H, Ar-H), 7.60-7.64 (d, 2H, Ar-H), 8.20 (s, 1H, CH), 10.70 (br, NH, $\mathrm{D}_{2} \mathrm{O}$ exchangeable)

3420 (br, NH), $2.72-2.78\left(\mathrm{~m}, 2 \mathrm{H}, \mathrm{CH}_{2}\right), 2.81-2.89\left(\mathrm{~m}, 2 \mathrm{H}, \mathrm{CH}_{2}\right), 3.84$, $1695(\mathrm{CO}), \quad 3.89\left(2 \mathrm{~s}, 6 \mathrm{H}, 2 \mathrm{OCH}_{3}\right), 6.94-7.00(\mathrm{~d}, 2 \mathrm{H}, \mathrm{Ar}-\mathrm{H}), 7.03-7.12$ $1595(\mathrm{C}=\mathrm{N}) \quad(\mathrm{d}, 2 \mathrm{H}, \mathrm{Ar}-\mathrm{H}), 7.16-7.22(\mathrm{~d}, 2 \mathrm{H}, \mathrm{Ar}-\mathrm{H}), 7.38-7.42(\mathrm{~d}, 2 \mathrm{H}$, Ar-H), 7.50-7.58 (d, 2H, Ar-H), 7.62-7.67 (d, 2H, Ar-H), 8.14 (s, 1H, CH), 11.00 (br, NH, $\mathrm{D}_{2} \mathrm{O}$ exchangeable)

3400 (br, NH), $2.74-2.80\left(\mathrm{~m}, 2 \mathrm{H}, \mathrm{CH}_{2}\right), 2.83-2.90\left(\mathrm{~m}, 2 \mathrm{H}, \mathrm{CH}_{2}\right), 3.82$, $1700(\mathrm{CO}), \quad 3.85,3.89\left(3 \mathrm{~s}, 9 \mathrm{H}, 3 \mathrm{OCH}_{3}\right), 6.90-6.96(\mathrm{~d}, 2 \mathrm{H}, \mathrm{Ar}-\mathrm{H})$, $1640(\mathrm{C}=\mathrm{N}) \quad 7.01-7.11(\mathrm{~d}, 2 \mathrm{H}, \mathrm{Ar}-\mathrm{H}), 7.15-7.20(\mathrm{~d}, 2 \mathrm{H}, \mathrm{Ar}-\mathrm{H})$, 7.36-7.40 (d, 2H, Ar-H), 7.52-7.57 (d, 2H, Ar-H), 7.59-7.65 (d, 2H, Ar-H), 8.18 (s, 1H, CH), 11.30 (br, NH, $\mathrm{D}_{2} \mathrm{O}$ exchangeable)

MS (m/z), $3385(\mathrm{br}, \mathrm{NH}), \quad 2.74-2.78\left(\mathrm{~m}, 2 \mathrm{H}, \mathrm{CH}_{2}\right), 2.86-2.94\left(\mathrm{~m}, 2 \mathrm{H}, \mathrm{CH}_{2}\right), 3.49$ (s, $\left.465\left(\mathrm{M}^{+}\right) \quad 1687(\mathrm{CO}), \quad 3 \mathrm{H}, \mathrm{N}-\mathrm{CH}_{3}\right), 3.84,3.90\left(2 \mathrm{~s}, 6 \mathrm{H}, 2 \mathrm{OCH}_{3}\right), 7.00(\mathrm{~d}, 2 \mathrm{H}, \mathrm{J}=$ $1615(\mathrm{C}=\mathrm{N}) \quad 8.38 \mathrm{~Hz}, \mathrm{Ar}-\mathrm{H}), 7.12(\mathrm{~d}, 2 \mathrm{H}, \mathrm{J}=8.42 \mathrm{~Hz}, \mathrm{Ar}-\mathrm{H}), 7.47$ (d, $2 \mathrm{H}, J=8.39 \mathrm{~Hz}, \mathrm{Ar}-\mathrm{H}), 7.58(\mathrm{~d}, 2 \mathrm{H}, J=8.41 \mathrm{~Hz}, \mathrm{Ar}-\mathrm{H})$, $8.19(\mathrm{~s}, 1 \mathrm{H}, \mathrm{CH}), 8.49$ (s, $1 \mathrm{H}$, triazole proton)

MS $(m / z), \quad 1688(\mathrm{CO}), \quad 2.23\left(\mathrm{~s}, 3 \mathrm{H}, \mathrm{CH}_{3}\right), 2.73-2.79\left(\mathrm{~m}, 2 \mathrm{H}, \mathrm{CH}_{2}\right), 2.83-2.90(\mathrm{~m}$, $\left.479\left(\mathrm{M}^{+}\right) \quad 1615(\mathrm{C}=\mathrm{N}) \quad 2 \mathrm{H}, \mathrm{CH}_{2}\right), 3.67\left(\mathrm{~s}, 3 \mathrm{H}, \mathrm{N}-\mathrm{CH}_{3}\right), 3.88,3.93(2 \mathrm{~s}, 6 \mathrm{H}$, $\left.2 \mathrm{OCH}_{3}\right), 7.07(\mathrm{~d}, 2 \mathrm{H}, \mathrm{J}=8.37 \mathrm{~Hz}, \mathrm{Ar}-\mathrm{H}), 7.14(\mathrm{~d}, 2 \mathrm{H}, \mathrm{J}=$ $8.38 \mathrm{~Hz}, \mathrm{Ar}-\mathrm{H}), 7.48$ (d, 2H, J = 8.40 Hz, Ar-H), 7.63 (d, $2 \mathrm{H}, J=8.40 \mathrm{~Hz}, \mathrm{Ar}-\mathrm{H}), 8.13(\mathrm{~s}, 1 \mathrm{H}, \mathrm{CH})$

3405 (br, NH), $2.35\left(\mathrm{~s}, 3 \mathrm{H}, \mathrm{CH}_{3}\right), 2.74-2.78\left(\mathrm{~m}, 2 \mathrm{H}, \mathrm{CH}_{2}\right), 2.84-2.91(\mathrm{~m}$, $\left.1685(\mathrm{CO}), \quad 2 \mathrm{H}, \mathrm{CH}_{2}\right), 3.86,3.90\left(2 \mathrm{~s}, 6 \mathrm{H}, 2 \mathrm{OCH}_{3}\right), 5.50\left(\mathrm{br}, \mathrm{NH}_{2}, \mathrm{D}_{2} \mathrm{O}\right.$ $1610(\mathrm{C}=\mathrm{N}) \quad$ exchangeable), $7.01(\mathrm{~d}, 2 \mathrm{H}, \mathrm{J}=8.41 \mathrm{~Hz}, \mathrm{Ar}-\mathrm{H}), 7.14(\mathrm{~d}$, $2 \mathrm{H}, J=8.40 \mathrm{~Hz}, \mathrm{Ar}-\mathrm{H}), 7.48(\mathrm{~d}, 2 \mathrm{H}, J=8.39 \mathrm{~Hz}, \mathrm{Ar}-\mathrm{H})$, $7.61(\mathrm{~d}, 2 \mathrm{H}, \mathrm{J}=8.40 \mathrm{~Hz}, \mathrm{Ar}-\mathrm{H}), 8.13$ (s, 1H, imidazole proton), $8.21(\mathrm{~s}, 1 \mathrm{H}, \mathrm{CH})$

MS (m/z). 3410 (brs, NH), 2.72-2.76 (m, 2H, $\left.\mathrm{CH}_{2}\right), 2.85-2.90\left(\mathrm{~m}, 2 \mathrm{H}, \mathrm{CH}_{2}\right), 3.87$, $541\left(\mathrm{M}^{+}\right) \quad 1686(\mathrm{CO}), \quad 3.91\left(2 \mathrm{~s}, 6 \mathrm{H}, 2 \mathrm{OCH}_{3}\right), 5.62\left(\mathrm{br}, \mathrm{NH}_{2}, \mathrm{D}_{2} \mathrm{O}\right.$ exchangeable), $1642(\mathrm{C}=\mathrm{N}) \quad 6.96-7.05(\mathrm{~m}, 4 \mathrm{H}, \mathrm{Ar}-\mathrm{H}), 7.15(\mathrm{~d}, 2 \mathrm{H}, J=8.42 \mathrm{~Hz}, \mathrm{Ar}-\mathrm{H})$, 7.34-7.43 (m, 3H, Ar-H), $7.50(\mathrm{~d}, 2 \mathrm{H}, J=8.41 \mathrm{~Hz}, \mathrm{Ar}-\mathrm{H})$, $7.62(\mathrm{~d}, 2 \mathrm{H}, \mathrm{J}=8.39 \mathrm{~Hz}, \mathrm{Ar}-\mathrm{H}), 8.09$ (s, 1H, imidazole proton), 8.19 (s, $1 \mathrm{H}, \mathrm{CH})$

MS (m/z), 3980 (brs, NH), 2.75-2.81 (m, 2H, $\left.\mathrm{CH}_{2}\right), 2.87-2.94\left(\mathrm{~m}, 2 \mathrm{H}, \mathrm{CH}_{2}\right), 3.88$, $529\left(\mathrm{M}^{+}\right) \quad 1687(\mathrm{CO}), \quad 3.93\left(2 \mathrm{~s}, 6 \mathrm{H}, 2 \mathrm{OCH}_{3}\right), 7.02(\mathrm{~d}, 2 \mathrm{H}, \mathrm{J}=8.39, \mathrm{~Hz}, \mathrm{Ar}-\mathrm{H})$, $1625(\mathrm{C}=\mathrm{N}) \quad 7.13-7.20(\mathrm{~m}, 4 \mathrm{H}, \mathrm{Ar}-\mathrm{H}), 7.30-7.38(\mathrm{~m}, 3 \mathrm{H}, \mathrm{Ar}-\mathrm{H}), 7.48$ $(\mathrm{d}, 2 \mathrm{H}, \mathrm{J}=8.38 \mathrm{~Hz}, \mathrm{Ar}-\mathrm{H}), 7.62(\mathrm{~d}, 2 \mathrm{H}, \mathrm{J}=8.40 \mathrm{~Hz}$, Ar-H), 8.19 (s, 1H, CH), 8.30 (s, 1H, azomethine proton), 9.00, 10.80 (br, 2H, 2NH, $\mathrm{D}_{2} \mathrm{O}$ exchangeable) 
$7 \mathbf{b}$

$\operatorname{MS}(m / z)$ $566\left(\mathrm{M}^{+}+2\right)$ $565\left(\mathrm{M}^{+}+1\right), \quad 1636(\mathrm{C}=\mathrm{N})$ $564\left(\mathrm{M}^{+}\right)$

7c

8 MS $(m / z)$ $452\left(\mathrm{M}^{+}\right)$

10a $\operatorname{MS}(\mathrm{m} / \mathrm{z})$, $508\left(\mathrm{M}^{+}\right)$

10b $\operatorname{MS}(\mathrm{m} / \mathrm{z})$, $505\left(\mathrm{M}^{+}\right)$ $426\left(\mathrm{M}^{+}\right)$ $2320\left(\mathrm{~N}_{3}\right)$, 1702 (CO), $1585(\mathrm{C}=\mathrm{N})$
3395 (br, NH), 2.71-2.77 (m, 2H, $\left.\mathrm{CH}_{2}\right), 2.80-2.86\left(\mathrm{~m}, 2 \mathrm{H}, \mathrm{CH}_{2}\right), 3.83$, $1678(\mathrm{CO}), \quad 3.88\left(2 \mathrm{~s}, 6 \mathrm{H}, 2 \mathrm{OCH}_{3}\right), 6.97(\mathrm{~d}, 2 \mathrm{H}, J=8.37 \mathrm{~Hz}, \mathrm{Ar}-\mathrm{H})$, $7.08(\mathrm{~d}, 2 \mathrm{H}, J=8.38 \mathrm{~Hz}, \mathrm{Ar}-\mathrm{H}), 7.26(\mathrm{~d}, 2 \mathrm{H}, J=8.40 \mathrm{~Hz}$, Ar-H), 7.51 (d, 2H, J = $8.41 \mathrm{~Hz}, \mathrm{Ar}-\mathrm{H}), 7.59$ (d, 2H, $J=$ $8.42 \mathrm{~Hz}, \mathrm{Ar}-\mathrm{H}), 7.69$ (d, 2H, $J=8.40 \mathrm{~Hz}, \mathrm{Ar}-\mathrm{H}), 8.06$ (s, $1 \mathrm{H}$, azomethine proton), 8.17 (s, $1 \mathrm{H}, \mathrm{CH}), 9.70,11.00$ (2brs, 2NH, $\mathrm{D}_{2} \mathrm{O}$ exchangeable)

3375 (brs, NH), 2.69-2.76 (m, 2H, $\left.\mathrm{CH}_{2}\right), 2.83-2.89\left(\mathrm{~m}, 2 \mathrm{H}, \mathrm{CH}_{2}\right), 3.79$, $1676(\mathrm{CO}), \quad 3.86,3.91\left(3 \mathrm{~s}, 9 \mathrm{H}, 3 \mathrm{OCH}_{3}\right), 7.09-7.10(\mathrm{~d}, 2 \mathrm{H}, \mathrm{Ar}-\mathrm{H})$, $1605(\mathrm{C}=\mathrm{N}) \quad$ 7.13-7.17 (d, 2H, Ar-H), 7.34-7.40 (d, 2H, Ar-H), 7.43-7.49 (d, 2H, Ar-H), 7.52-7.57 (d, 2H, Ar-H), 7.65-7.70 (d, 2H, Ar-H), 8.05 (s,1H, azomethine proton), 8.17 (s, 1H, CH), 9.25, 10.30 (brs, 2NH, $\mathrm{D}_{2} \mathrm{O}$ exchangeable)

$9 \operatorname{MS}(m / z)$

3340 (br, NH), $1625(\mathrm{~N}=\mathrm{N})$, 2.71-2.78 (m, 2H, $\left.\mathrm{CH}_{2}\right), 2.82-2.90\left(\mathrm{~m}, 2 \mathrm{H}, \mathrm{CH}_{2}\right), 3.86$, $3.90\left(2 \mathrm{~s}, 6 \mathrm{H}, 2 \mathrm{OCH}_{3}\right), 6.93-6.98(\mathrm{~d}, 2 \mathrm{H}, \mathrm{Ar}-\mathrm{H}), 7.11-7.17$ (d, 2H, Ar-H), 7.37-7.42 (d, 2H, Ar-H), 7.56-7.60 (d, 2H, Ar-H), 8.21 (s, 1H, CH), 11.60 (br, NH, $\mathrm{D}_{2} \mathrm{O}$ exchangeable)

3410 (br, NH), 2.75-2.82 (m, 2H, $\left.\mathrm{CH}_{2}\right), 2.84-2.93\left(\mathrm{~m}, 2 \mathrm{H}, \mathrm{CH}_{2}\right), 3.86$, $1687(\mathrm{CO}), \quad 3.89\left(2 \mathrm{~s}, 6 \mathrm{H}, 2 \mathrm{OCH}_{3}\right), 7.03-7.10(\mathrm{~d}, 2 \mathrm{H}, \mathrm{Ar}-\mathrm{H}), 7.16-7.22$ $1589(\mathrm{C}=\mathrm{N}) \quad(\mathrm{d}, 2 \mathrm{H}, \mathrm{Ar}-\mathrm{H}), 7.38-7.42(\mathrm{~d}, 2 \mathrm{H}, \mathrm{Ar}-\mathrm{H}), 7.58-7.64(\mathrm{~d}, 2 \mathrm{H}$, $\mathrm{Ar}-\mathrm{H}), 8.23$ (s, 1H, CH), 8.56 (br, 2H, $\mathrm{NH}_{2}$ ), 11.30 (br, $\mathrm{NH})\left(\mathrm{NH}_{2}, \mathrm{NH}, \mathrm{D}_{2} \mathrm{O}\right.$ exchangeable)

3480 (br, OH), 2.74-2.82 (m, 2H, $\left.\mathrm{CH}_{2}\right), 2.86-2.93\left(\mathrm{~m}, 2 \mathrm{H}, \mathrm{CH}_{2}\right), 3.55$ (br, 3318 (brs, $\mathrm{NH}), 1 \mathrm{H}, \mathrm{OH}$ coupled with $\mathrm{H}_{2} \mathrm{O}$ of DMSO), 3.85, 3.90 (2s, $6 \mathrm{H}$, 1687, (CO), $\left.\quad 2 \mathrm{OCH}_{3}\right), 5.67\left(\mathrm{br}, \mathrm{NH}_{2}, \mathrm{D}_{2} \mathrm{O}\right.$ exchangeable), 6.68 (s, 1H, $1601(\mathrm{C}=\mathrm{N}) \quad$ pyrazole proton), $7.00(\mathrm{~d}, 2 \mathrm{H}, \mathrm{J}=8.41 \mathrm{~Hz}, \mathrm{Ar}-\mathrm{H}), 7.26$ (d, $2 \mathrm{H}, J=8.40 \mathrm{~Hz}, \mathrm{Ar}-\mathrm{H}), 7.34(\mathrm{~d}, 2 \mathrm{H}, J=8.40 \mathrm{~Hz}$, Ar-H), 7.53 (d, 2H, J = 8.40 Hz, Ar-H), 8.14 (s, 1H, CH), 9.10 (brs, $\mathrm{NH}, \mathrm{D}_{2} \mathrm{O}$ exchangeable)

3380 (br, NH), $2.21\left(\mathrm{~s}, 3 \mathrm{H}, \mathrm{CH}_{3}\right), 2.68-2.73\left(\mathrm{~m}, 2 \mathrm{H}, \mathrm{CH}_{2}\right), 2.86(\mathrm{~s}, 3 \mathrm{H}$, $\left.1690(\mathrm{CO}), \quad \mathrm{CH}_{3}\right), 2.89-2.96\left(\mathrm{~m}, 2 \mathrm{H}, \mathrm{CH}_{2}\right), 3.85,3.89(2 \mathrm{~s}, 6 \mathrm{H}$, $\left.1625(\mathrm{C}=\mathrm{N}) \quad 2 \mathrm{OCH}_{3}\right), 6.25(\mathrm{~s}, 1 \mathrm{H}$, pyrazole proton $), 7.11(\mathrm{~d}, 2 \mathrm{H}, J=$ $8.38 \mathrm{~Hz}, \mathrm{Ar}-\mathrm{H}), 7.31$ (d, 2H, J = 8.39 Hz, Ar-H), 7.45 (d, $2 \mathrm{H}, J=8.40 \mathrm{~Hz}, \mathrm{Ar}-\mathrm{H}), 7.64(\mathrm{~d}, 2 \mathrm{H}, J=8.40 \mathrm{~Hz}, \mathrm{Ar}-\mathrm{H})$, 8.17 (s, 1H, CH), 10.20 (br, NH, $\mathrm{D}_{2} \mathrm{O}$ exchangeable)

10c $\quad$ MS $(m / z), \quad 3380(\mathrm{br}, \mathrm{NH})$, $542\left(\mathrm{M}^{+}+2\right), 1690(\mathrm{CO})$, $541\left(\mathrm{M}^{+}+1\right), \quad 1625(\mathrm{C}=\mathrm{N})$ $540\left(\mathrm{M}^{+}\right)$

11 MS $(m / z)$, $553\left(\mathrm{M}^{+}\right)$ $2.19\left(\mathrm{~s}, 3 \mathrm{H}, \mathrm{CH}_{3}\right), 2.67-2.74\left(\mathrm{~m}, 2 \mathrm{H}, \mathrm{CH}_{2}\right), 2.87$ (s, 3H, $\left.\mathrm{CH}_{3}\right), 2.90-2.97\left(\mathrm{~m}, 2 \mathrm{H}, \mathrm{CH}_{2}\right), 3.86,3.90(2 \mathrm{~s}, 6 \mathrm{H}$, $\left.2 \mathrm{OCH}_{3}\right), 7.12(\mathrm{~d}, 2 \mathrm{H}, J=8.39 \mathrm{~Hz}, \mathrm{Ar}-\mathrm{H}), 7.33(\mathrm{~d}, 2 \mathrm{H}, J=$ $8.39 \mathrm{~Hz}, \mathrm{Ar}-\mathrm{H}), 7.47$ (d, 2H, $=8.41 \mathrm{~Hz}, \mathrm{Ar}-\mathrm{H}), 7.65$ (d, $2 \mathrm{H}, J=8.41 \mathrm{~Hz}, \mathrm{Ar}-\mathrm{H}), 8.09$ (s, 1H, CH), 10.35 (br, NH, $\mathrm{D}_{2} \mathrm{O}$ exchangeable)

3250 (br, NH), $1.24-1.31\left(\mathrm{t}, 3 \mathrm{H}, \mathrm{CH}_{3}\right), 2.15\left(\mathrm{~s}, 3 \mathrm{H}, \mathrm{CH}_{3}\right), 2.71-2.78(\mathrm{~m}$, 1730, $\left.16852 \mathrm{H}, \mathrm{CH}_{2}\right), 2.85-2.92\left(\mathrm{~m}, 2 \mathrm{H}, \mathrm{CH}_{2}\right), 3.42\left(\mathrm{~s}, 2 \mathrm{H}, \mathrm{CH}_{2}\right)$, (2CO), $3.84,3.86\left(2 \mathrm{~s}, 6 \mathrm{H}, 2 \mathrm{OCH}_{3}\right), 4.00-4.10\left(\mathrm{q}, 2 \mathrm{H}, \mathrm{CH}_{2}\right), 7.01$ $(\mathrm{d}, 2 \mathrm{H}, J=8.39 \mathrm{~Hz}, \mathrm{Ar}-\mathrm{H}), 7.13(\mathrm{~d}, 2 \mathrm{H}, \mathrm{J}=8.40 \mathrm{~Hz}$, Ar-H), 7.45 (d, 2H, J = 8.39 Hz, Ar-H), $7.62(\mathrm{~d}, 2 \mathrm{H}, J=$ $8.41 \mathrm{~Hz}, \mathrm{Ar}-\mathrm{H}), 8.17$ (s, 1H, CH), 9.40, 11.00 (2br, 2NH, $\mathrm{D}_{2} \mathrm{O}$ exchangeable) 
H. N. Hafez et al.: Synthesis and evaluation of analgesic, anti-inflammatory and ulcerogenic activities of some triazolo- and 2-pyrazolyl-pyrido[2,3-d]-pyrimidines, Acta Pharm. 58 (2008) 359-378.

12

$\operatorname{MS}(m / z)$ $507\left(\mathrm{M}^{+}\right)$

$\mathrm{MS},\left[\mathrm{M}^{+}\right]$, $m / z 482$ $(100 \%)$
3400 (brs, NH), 2.03 (s, 3H, $\left.\mathrm{CH}_{3}\right), 2.78-2.83\left(\mathrm{~m}, 2 \mathrm{H}, \mathrm{CH}_{2}\right), 2.86-2.92(\mathrm{~m}$, 1697,1684 (2CO), $1550(\mathrm{C}=\mathrm{N})$ $\left.2 \mathrm{H}, \mathrm{CH}_{2}\right), 3.87,3.92\left(2 \mathrm{~s}, 6 \mathrm{H}, 2 \mathrm{OCH}_{3}\right), 4.31\left(\mathrm{~s}, 2 \mathrm{H}, \mathrm{CH}_{2}\right)$, $7.06(\mathrm{~d}, 2 \mathrm{H}, \mathrm{J}=8.36 \mathrm{~Hz}, \mathrm{Ar}-\mathrm{H}), 7.35(\mathrm{~d}, 2 \mathrm{H}, J=8.38 \mathrm{~Hz}$, Ar-H), $7.45(\mathrm{~d}, 2 \mathrm{H}, J=8.39 \mathrm{~Hz}, \mathrm{Ar}-\mathrm{H}), 7.70(\mathrm{~d}, 2 \mathrm{H}, J=$ $8.38 \mathrm{~Hz}, \mathrm{Ar}-\mathrm{H}), 8.23$ (s, 1H, CH), 10.30 (brs, NH, $\mathrm{D}_{2} \mathrm{O}$ exchangeable)

3465 (br, NH), $2.79-2.85\left(\mathrm{~m}, 2 \mathrm{H}, \mathrm{CH}_{2}\right), 2.88-2.93\left(\mathrm{~m}, 2 \mathrm{H}, \mathrm{CH}_{2}\right), 3.88$, $1686(\mathrm{CO}), \quad 3.93\left(2 \mathrm{~s}, 6 \mathrm{H}, 2 \mathrm{OCH}_{3}\right), 7.07(\mathrm{~d}, 2 \mathrm{H}, \mathrm{J}=8.38 \mathrm{~Hz}, \mathrm{Ar}-\mathrm{H})$, $1620(\mathrm{C}=\mathrm{N})$ $7.38(\mathrm{~d}, 2 \mathrm{H}, J=8.39 \mathrm{~Hz}, \mathrm{Ar}-\mathrm{H}), 7.48(\mathrm{~d}, 2 \mathrm{H}, J=8.41 \mathrm{~Hz}$, Ar-H), 7.68 (d, 2H, J = 8.42 Hz, Ar-H), $8.16(\mathrm{~s}, 1 \mathrm{H}, \mathrm{CH})$, $10.00,11.50$ (2brs, 2NH, $\mathrm{D}_{2} \mathrm{O}$ exchangeable)

$\mathrm{cm}^{-1}$ (N-H stretching). Also, ${ }^{1} \mathrm{H}$ NMR spectra of compounds $4 \mathbf{a}, \mathbf{b}$ showed broad bands at $\delta 9.50$ and $10.20 \mathrm{ppm}$, while IR and NMR spectra of compound $\mathbf{5 a}, \mathbf{b}$ revealed the absence of $\mathrm{NH}$ absorption peaks (Table II).

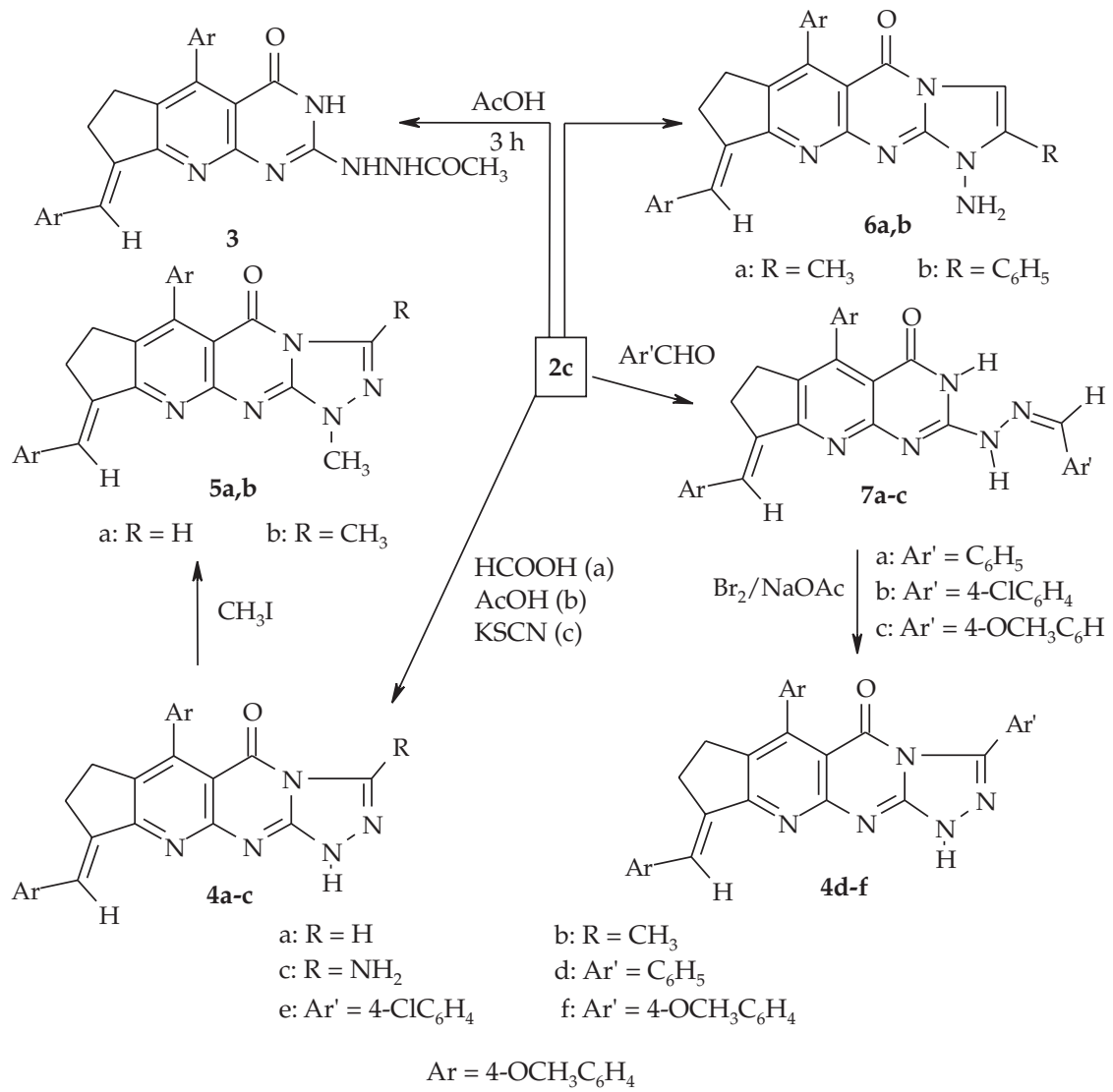

Scheme 2 
Also, the 2-hydrazino- derivative $2 \mathrm{c}$ reacted with potassium thiocyanate in boiling acetic acid to give 3-amino-6-(4-methoxyphenyl)-9-(4-methoxyphenylmethylene)-1,7,8, 9-tetrahydro-cyclopenta[5,6]pyrido[2,3- $d][1,2,4]$ triazolo[4,3-a]pyrimidin-5(H)-one $(4 \mathrm{c})$. Beside correct values in elemental analyses, the spectral data of $4 \mathrm{c}$ are in agreement with the assigned structure (Scheme 2). When compound 2c was heated under reflux with $\alpha$-haloketones, namely, chloroacetone or 2-bromoacetophenone in dry xylene, it yielded the respective 1-amino-6-aryl-9-arylidene- $1 H, 5 H$-imidazo[1',2'-a]cyclopenta[5,6]pyrido$-[2,3-d]$ pyrimidin-5-one $(6 \mathbf{6}, \mathbf{b})$. The ${ }^{1} \mathrm{H}$ NMR spectrum of $\mathbf{6 a}$, for example, showed that singlet signals at 2.35 ppm supported the methyl protons, two signals around 2.74-2.91 ppm corresponded to the two methylene groups, two singlets around 3.85-3.90 ppm due to the two methoxy groups and the broad absorption signal $\left(\mathrm{D}_{2} \mathrm{O}\right.$ exchangeable) supported the amino group. In addition to the pyrazole proton at $8.13 \mathrm{ppm}$, the spectrum showed four doublet signals due to the $p$-substituted phenyl groups with the coupling constant around $8.40 \mathrm{~Hz}$ and the methylenic proton at $8.21 \mathrm{ppm}$. Also, the ${ }^{13} \mathrm{C}$ NMR showed that, in addition to the methyl group (18.39 ppm), two methylene groups (27.21 and $27.90 \mathrm{ppm}$ ), two methoxy groups (54.97 and $55.33 \mathrm{ppm}$ ) and carbonyl groups (163.8 ppm), eighteen lines around $111.0-158.5 \mathrm{ppm}$ corresponding to $22 \mathrm{sp}^{2}$ carbon atoms were obtained (Table III).

Table III. ${ }^{13} \mathrm{C}$ NMR of some new compounds

\begin{tabular}{|c|c|}
\hline $\begin{array}{l}\text { Compd. } \\
\text { No. }\end{array}$ & 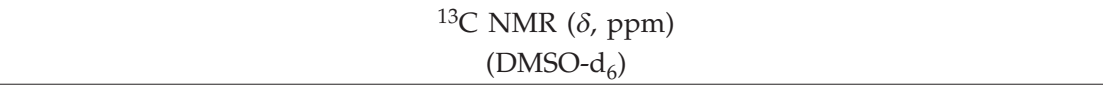 \\
\hline $2 c$ & $\begin{array}{l}27.01,27.95\left(2 \mathrm{C}, 2 \mathrm{CH}_{2}\right), 54.98,55.17,\left(2 \mathrm{C}, 2 \mathrm{OCH}_{3}\right), 108.5,112.9,113.1,114.2,124.4 \\
128.9,129.5,130.6,130.7,130.8,132.9,138.9,148.2,154.7,158.3,158.8 \text { (16 line for } 20 \\
\text { sp }^{2} \text { carbon atoms) and } 163.9(\mathrm{CO})\end{array}$ \\
\hline $4 a$ & $\begin{array}{l}27.12,27.93\left(2 \mathrm{C}, 2 \mathrm{CH}_{2}\right), 54.95,55.21,\left(2 \mathrm{C}, 2 \mathrm{OCH}_{3}\right), 110.2,112.9,113.8,118.5,125.4 \text {, } \\
127.3,129.1,131.7,132.3,133.8,134.9,137.6,147.5,153.4,155.3,157.2,158.3 \text { (17 line } \\
\text { for } 21 \mathrm{sp}^{2} \text { carbon atoms) and } 165.2(\mathrm{CO})\end{array}$ \\
\hline $4 c$ & $\begin{array}{l}{ }^{13} \mathrm{C}-\mathrm{NMR}: 27.11,27.96\left(2 \mathrm{C}, 2 \mathrm{CH}_{2}\right), 54.96,55.18,\left(2 \mathrm{C}, 2 \mathrm{OCH}_{3}\right), 110.4,112.3,113.6,118.2 \text {, } \\
124.5,127.9,128.4,129.3,130.7,130.2,131.8,134.9,138.5,148.1,154.4,158.2,158.8(17 \\
\text { line for } 21 \mathrm{sp}^{2} \text { carbon atoms) and } 163.5(\mathrm{CO})\end{array}$ \\
\hline $4 f$ & $\begin{array}{l}\text { 27.11, 27.94 }\left(2 \mathrm{C}, 2 \mathrm{CH}_{2}\right), 54.96,55.15,56.23\left(3 \mathrm{C}, 3 \mathrm{OCH}_{3}\right), 108.3,112.5,113.5,114.7, \\
124.7,128.6,129.5,130.9,131.2,131.8,132.3,132.7,134.8,138.9,140.2,144.6,148.2, \\
154.7,167.0,158.3,158.8\left(21 \text { line for } 27 \mathrm{sp}^{2} \text { carbon atoms }\right) \text { and } 163.9(\mathrm{CO})\end{array}$ \\
\hline $6 a$ & $\begin{array}{l}18.39\left(\mathrm{CH}_{3}\right), 27.21,27.90\left(2 \mathrm{C}, 2 \mathrm{CH}_{2}\right), 54.97,55.33,\left(2 \mathrm{C}, 2 \mathrm{OCH}_{3}\right), 111.0,112.7,114.2, \\
118.6,125.7,127.7,128.5,129.5,131.3,132.5,133.8,135.6,137.8,147.4,152.9,154.3, \\
157.4,158.5\left(18 \text { line for } 22 \mathrm{sp}^{2} \text { carbon atoms) and } 163.8(\mathrm{CO})\right.\end{array}$ \\
\hline 13 & $\begin{array}{l}\text { 24.31, } 26.58\left(2 \mathrm{CH}_{2}\right), 109.3,127.0,127.5,127.6,127.7,127.8,129.2,129.4,130.9,132.1, \\
\text { 133.1, 136.6, 138.7, } 150.5,153.7,158.4\left(16 \text { line for } 20 \mathrm{sp}^{2} \text { carbon atoms), } 163.8(\mathrm{CO})\right. \\
\text { and } 174.2(\mathrm{C}=\mathrm{S})\end{array}$ \\
\hline
\end{tabular}

Further, compound $2 \mathrm{c}$ gave the 2-arylmethylene hydrazone derivatives $7 \mathrm{a}-\mathrm{c}$ when treated with the appropriate aldehyde in boiling glacial acetic acid. The latter compounds were cyclized when gently heated in a mixture of bromine and sodium acetate in acetic acid to afford 3-aryltriazolo $\left[4^{\prime}, 3^{\prime}: 1,2\right]$ cyclopentenopyrido[2,3-d]pyrimidines (4d-f). Beside 
correct values in elemental analysis, the spectral data of $7 \mathbf{a}-\mathbf{c}$ and $4 \mathbf{d}-\mathbf{f}$ are in agreement with the assigned structures. The ${ }^{1} \mathrm{H}$ NMR spectra revealed the absence of azomethine protons. Also, the formation of triazolopyridopyrimidine $4 \mathrm{~d}-\mathrm{f}$ from $7 \mathrm{a}-\mathrm{c}$ may be accomplished via brominating the methylenic proton or N-3 of the pyrimidine ring followed by elimination of hydrogen bromide, as shown in Scheme 3.

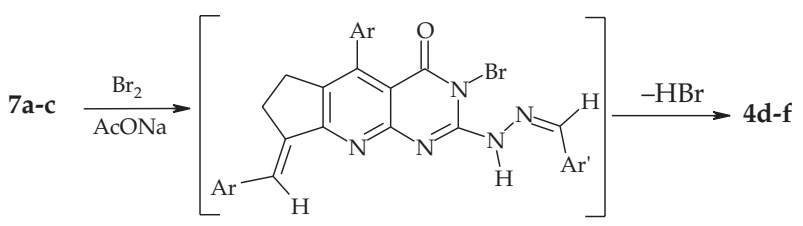

Scheme 3

Treatment of compound $2 \mathrm{c}$ with nitrous acid led to the formation of 6-(4-methoxyphenyl)-9-(4-methoxyphenylmethylene)-1,7,8,9-tetrahydrocyclopenta[5,6]-pyrido[2,3- $d]$ [1, 2,3,4] tetrazolo[4,5-a]pyrimidin-5(H)-one (8) (Scheme 4). The IR spectrum of 8 displayed absorption bands at $3240 \mathrm{~cm}^{-1}(\mathrm{NH})$ and $1702 \mathrm{~cm}^{-1}(\mathrm{CO})$. The latter compound was reduced to 2-amino-5-(4-methoxyphenyl)-8-(4-methoxyphenyl-methylene)-7,8-dihydro$-6 H$-cyclopenta[5,6]pyrido[2,3- $d]$ pyrimidine- $4(H)$-one $(9)$ by zinc dust and acetic acid.

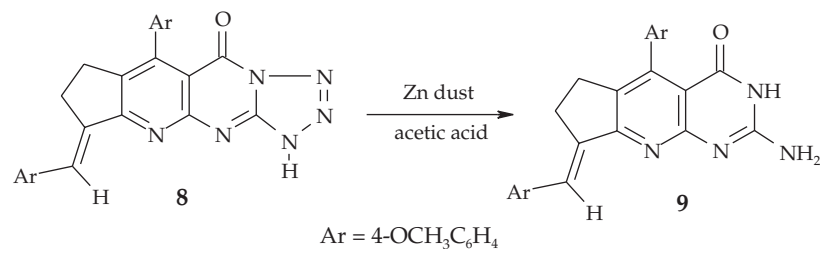

Scheme 4

5-(4-Methoxyphenyl)-8-(4-methoxyphenylmethylene)-2-hydrazino-7,8-dihydro-6H-cyclopenta $[5,6]$ pyrido $2,3-d]$ pyrimidin-4(H)-one (2c) reacted with $\beta$-cyano-ester, $\beta$-diketones and $\beta$-ketoesters to form 2-(1-pyrazolyl) derivatives. Thus, heating compound $2 \mathrm{c}$ with ethylcyanoacetate, pentane-2,4-dione and/or 3-chloropentane-2,4-dione, yielded the respective 2-(3,5-substituted-4-(un)substituted-pyrazol-1-yl)-5-(4-methoxyphenyl)-8-(4-methoxy-phenyl-methylene)-7,8-dihydro-6H-cyclopenta[5,6]pyrido[2,3- $d]$ pyrimidin-4(H)-ones 10a-c, respectively (Scheme 5).

Compound $2 \mathrm{c}$ condensed with ethyl acetoacetate upon heating in boiling ethanol to afford 2-[ethyl-5-(4-methoxyphenyl)-8-(4-methoxyphenylmethylene)-7,8-dihydro-6H-cyclopenta[5,6]pyrido[2,3-d]pyrimidin-4(H)-one]acetatehydrazone (11), which could be cyclized either by prolonged heating in ethanol or by heating in sodium ethoxide solution to give 5-(4-methoxyphenyl)-8-(4-methoxyphenylmethylene)-2-(3-methyl-4H-pyrazol-5-one-1-yl)-7,8-dihydro-6H-cyclopenta[5,6]pyrido[2,3-d]pyrimidin-4(H)-one (12).

Finally, heating $2 \mathrm{c}$ with carbon disulphide in ethanolic potassium hydroxide solution gave 3-thioxo-6-(4-methoxyphenyl)-9-(methoxyphenylmethylene)-1,2,8,9-tetrahydro- 
H. N. Hafez et al.: Synthesis and evaluation of analgesic, anti-inflammatory and ulcerogenic activities of some triazolo- and 2-pyrazolyl-pyrido[2,3-d]-pyrimidines, Acta Pharm. 58 (2008) 359-378.<smiles></smiles>

Scheme 5

Table IV. Percent inflammatory activity of the tested compounds (carrageenean-induced paw odema test in rats)

\begin{tabular}{cccc}
\hline \multirow{2}{*}{$\begin{array}{c}\text { Compd. } \\
\text { No. }\end{array}$} & \multicolumn{3}{c}{ Inhibition $(\%)^{\mathrm{a}}$} \\
\cline { 2 - 4 } & 3 hours & 4 hours & Potency \\
\hline $\mathbf{2 a}$ & $27.29 \pm 2.64$ & $28.27 \pm 2.75^{\mathrm{b}}$ & 0.34 \\
$\mathbf{2 b}$ & $56.82 \pm 2.53$ & $56.81 \pm 2.16^{\mathrm{b}}$ & 0.71 \\
$\mathbf{2 c}$ & $52.28 \pm 2.18$ & $54.55 \pm 2.62^{\mathrm{b}}$ & 0.67 \\
$\mathbf{4 a}$ & $27.28 \pm 2.73$ & $29.54 \pm 2.79^{\mathrm{b}}$ & 0.37 \\
$\mathbf{4 c}$ & $75.01 \pm 2.52$ & $77.26 \pm 1.92^{\mathrm{b}}$ & 0.98 \\
$\mathbf{6 a}$ & $63.70 \pm 1.93$ & $63.62 \pm 1.92$ & 0.80 \\
$\mathbf{9}$ & $75.00 \pm 2.79$ & $79.53 \pm 3.72$ & 1.00 \\
$\mathbf{1 0 a}$ & $76.08 \pm 2.67$ & $82.79 \pm 2.18$ & 1.03 \\
$\mathbf{1 0 b}$ & $31.80 \pm 3.45$ & $31.80 \pm 3.69^{\mathrm{b}}$ & 0.39 \\
$\mathbf{1 1}$ & $15.90 \pm 2.73$ & $19.04 \pm 2.24$ & 0.24 \\
$\mathbf{1 2}$ & $52.27 \pm 2.80$ & $52.27 \pm 2.79^{\mathrm{b}}$ & 0.65 \\
$\mathbf{1 3}$ & $56.81 \pm 1.94$ & $59.09 \pm 2.74^{\mathrm{b}}$ & 0.74 \\
Control & - & - & - \\
Ibuprofen & $75.00 \pm 2.53$ & $79.54 \pm 2.25$ & 1.00 \\
\hline
\end{tabular}

Dose: $30 \mathrm{mg} \mathrm{kg}^{-1} \mathrm{~b} . \mathrm{m}$. of the tested compound and standard drug.

a Mean \pm SEM $(n=6)$.

b Significant difference relative to ibuprofen: $p<0.01$. 
-7H-cyclopenta[5,6]pyrido[2,3- $d][1,2,4]$ triazolo[4,3-a]pyrimidin-5(H)-one (13) (Scheme 5). The IR spectrum of 13 displayed absorption bands at $3465-3400 \mathrm{~cm}^{-1}(2 \mathrm{NH})$ and 1686 $\mathrm{cm}^{-1}(\mathrm{CO})$. The ${ }^{13} \mathrm{C}$ NMR spectrum $\left(\mathrm{DMSO}-\mathrm{d}_{6}\right.$ ) of $\mathbf{1 3}$ showed two signals due to the $\mathrm{sp}^{3}$ carbon atoms, sixteen lines assigned to $20 \mathrm{sp}^{2}$ carbon atoms and, in addition to the signal for the carbonyl group, the spectrum showed a strong peak corresponding to $C=S$ at $174.2 \mathrm{ppm}$.

The pharmacological screening of the tested compounds showed anti-inflammatory activity ranging from 19.0 to $82.8 \%$ (Table IV), whereas the standard drug ibuprofen showed $79.5 \%$ inhibition after $4 \mathrm{~h}$. The anti-inflammatory activity of 2-pyrazolyl-pyridopyrimidine derivatives $\mathbf{1 0 a}, \mathbf{1 0 b}$ and $\mathbf{1 2}$ ranged from 31.8 to $82.8 \%$. The aminopyrazolyl derivative 10a with hydroxyl and amino groups showed higher activity than the standard drug ibuprofen, whereas when these groups were replaced by a methyl group, the anti-inflammatory activity decreased. Also, it was observed that the triazolopyrido-pyrimidine derivatives $\mathbf{4 a}, \mathbf{4 c}$ and $\mathbf{1 3}$ showed activity ranging from 29.5 to $77.3 \%$, and 3-aminotriazolopyridopyrimidine $4 \mathrm{c}$ showed activity nearly equivalent to the standard drug. 2-Aminopyrido[2,3-d]pyrimidine (9) showed activity (79.5\%) equivalent to that of ibuprofen. Other tested compounds showed moderate anti-inflammatory activity. It is clear from Table IV that the presence of amino group in triazolo-/or pyrazolo-pyridopyrimidine increases the anti-inflammatory activity.

Compounds 4c, 6a, 9 and 10a which showed anti-inflammatory activity comparable or equal to that of the standard were further tested for their analgesic activity at a dose of $30 \mathrm{mg} \mathrm{kg}^{-1}$ ibuprofen (Table V). Compounds showed analgesic activity ranging from 58.4 to $72.7 \%$, whereas the standard drug ibuprofen showed $69.5 \%$ inhibition. Compounds 4c, 9 and 10a showed the highest anti-inflammatory activity, 72.7, 59.3 and $70.2 \%$, resp. The remaining compounds showed reduced analgesic activity. These compounds were further screened for their acute ulcerogenic activity. The tested compounds showed significant reduction in ulcerogenic activity ranging from $0.7 \pm 0.15$ to $1.06 \pm 0.17$, whereas

Table V. Analgesic and ulcerogenic activity of the selected compounds

\begin{tabular}{cccccc}
\hline \multirow{2}{*}{ Group } & \multicolumn{5}{c}{ Analgesic activity } \\
\cline { 2 - 6 } & $\begin{array}{c}\text { Pre-treatment } \\
\text { normal }(0 \mathrm{~h})\end{array}$ & $\begin{array}{c}\text { Post-treatment } \\
\text { after }(4 \mathrm{~h})\end{array}$ & $\begin{array}{c}\text { Inhibition } \\
(\%)^{\mathrm{a}}\end{array}$ & Potency & $\begin{array}{c}\text { Ulcerogenic } \\
\text { index }\end{array}$ \\
\hline $\mathbf{4 c}$ & $1.40 \pm 0.150$ & $2.42 \pm 0117^{\mathrm{b}}$ & 72.7 & 1.04 & $1.064 \pm 0.17^{\mathrm{c}}$ \\
$\mathbf{6 a}$ & $1.34 \pm 0.137$ & $2.13 \pm 0145^{\mathrm{b}}$ & 58.4 & 0.84 & $0.670 \pm 0.21^{\mathrm{c}}$ \\
$\mathbf{9}$ & $1.34 \pm 0.136$ & $2.12 \pm 0.146^{\mathrm{b}}$ & 59.3 & 0.85 & $0.732 \pm 0.18^{\mathrm{c}}$ \\
$\mathbf{1 0 a}$ & $1.41 \pm 0.150$ & $1.86 \pm 0.094^{\mathrm{b}}$ & 70.2 & 1.02 & $0.667 \pm 0.15^{\mathrm{c}}$ \\
$\mathbf{1 3}$ & $1.80 \pm 0.188$ & $2.35 \pm 0.180^{\mathrm{b}}$ & 31.2 & 1.02 & $0.668 \pm 0.22^{\mathrm{c}}$ \\
Ibuprofen & $1.15 \pm 0.060$ & $1.95 \pm 0.097$ & 69.5 & 1.00 & $1.665 \pm 0.25$ \\
Control & - & - & & & - \\
\hline
\end{tabular}

Dose: $30 \mathrm{mg} \mathrm{kg}^{-1} \mathrm{~b} . \mathrm{m}$. of the tested compound and standard drug.

a Mean $\pm \operatorname{SEM}(n=6)$.

b Significant difference relative to ibuprofen: $p<0.0001$.

c Significant difference from ibuprofen: $p<0.05$. 
H. N. Hafez et al.: Synthesis and evaluation of analgesic, anti-inflammatory and ulcerogenic activities of some triazolo- and 2-pyrazolyl-pyrido[2,3-d]-pyrimidines, Acta Pharm. 58 (2008) 359-378.

the standard drug ibuprofen showed a severity index of $1.66 \pm 0.25$. Maximum reduction in ulcerogenic activity was found for compound 10a having the hydroxyl and amino groups of 2-pyrazolo-pyridopyrimidine ring $(0.66 \pm 0.15)$. The rest of the compounds also showed a better gastro interact safety profile than ibuprofen.

\section{CONCLUSIONS}

The present investigation offers new, rapid and effective procedures for the synthesis of new poly-condensed heterocyclic pyrido[2,3-d]pyrimidine ring systems. Compounds 3-amino-6-(4-methoxyphenyl)-9-(4-methoxyphenylmethylene)-cyclopenta-[5,6]pyrido $[2,3-d][1,2,4]$ triazolo[ $4,3-a]$ pyrimidin-5(H)-one (4c), 1-amino-2-methyl-6-(4-methoxyphenyl)-9-(4-methoxyphenylmethylene)-cyclopenta[5,6]pyrido[2,3- $d$ ] imidazolo-[1,2-a]pyrimidin-5 (H)-one (6a) and 2-amino-5-(4-methoxy-phenyl)-8-(4-methoxyphenyl-methylene)-7,8-dihydro-6H-cyclopenta[5,6]pyrido[2,3-d]pyrimidin-4(H)-one (9) exhibited a potent anti-inflammatory activity whereas 2-(3-amino-5-hydroxypyrazol-1-yl)-pyrido[2,3-d]pyrimidin-4-one derivative 10a was the most active.

Acknowledgements. - The authors are thankful to the National Research Center for providing laboratory facilities, Micro-analytical Centre, Cairo University and the Pharmacological Unit National Research Centre, for microanalyses and pharmacological screening of the compounds.

\section{REFERENCES}

1. A. B. A. El-Gazzar, M. M. El-Enany and M. N. Mahmoud, Synthesis, Analgesic, anti-inflammatory and antimicrobial activity of some novel pyrimido[4,5-d]quinolin-4-ones, Bioorg. Med. Chem. 16 (2008) 3261-3273; DOI: 10.1016/j.bmc.2007.12.012.

2. D. H. Boschelli, Z. Wu, S. R. Klutchko, H. D. Showalter, J. M. Hamby, G. H. Lu, T. C. Major, T. K. Dahring, B. Batley, R. L. Panek, J. Keiser, B. G. Hartl, A. J. Kraker, W. D. Klohs, B. J. Roberts, S. Patmore, W. L. Elliott, R. Steinkampf, L. A. Bradford, H. Hallak and A. M. Doherty, Synthesis and tyrosine kinase inhibitory activity of a series of 2-amino- $8 H$-pyrido[2,3- $d$ ]pyrimidines: identification of potent selective platelet-derived growth factor receptor tyrosine kinase inhibitors, J. Med. Chem. 41 (1998) 4365-4377; DOI: 10.1021/jm980398y 50022-2623(98)00398-7.

3. S. R. Natarajan, D. D. Wisnoski, J. E. Thompson, E. A. O'Neill and S. J. O'Keefe, p38 MAP kinase inhibitors. Part 3: SAR on 3,4-dihydropyrimido[4,5-d]pyrimidin-2-ones and 3,4-dihydropyrido[4,3-d]pyrimidin-2-ones, Bioorg. Med. Chem. Lett. 16 (2006) 4400-4404; DOI: 10.1016/j.bmcl. 2006.05.045.

4. C. B. Vu, G. P. Luke, N. Kawahata, W. C. Shakespeare, Y. Wang, R. Sundara-Moorthi, C. A. Metcalf, T. P. Keenan, S. Pradeepan, E. Corpuz, T. Merry, R. S. Bohacek, D. C. Dalgarno, S. S. Narula, M. R. van Schravendijk, M. K. Ram, S. Adams, S. Liou, J. A. Keats, S. M. Violette, W. Guan, M. Weigele and T. K. Sawyer, Bone-targeted pyrido[2,3- $d]$ pyrimidin-7-ones: potent inhibitors of src tyrosine kinase as novel antiresorptive agents, Bioorg. Med. Chem. Lett. 13 (2003) 3071-3074; DOI: 10.1016/S0960-894X(03)00649-8.

5. A. J. Kraker, B. G. Hartl, A. M. Amar, M. R. Barvian, H. D. Hollis Showalter and C. W. Moore, Biochemical and cellular effect of c-Src kinase-selective pyrido[2,3- $d]$ pyrimidine tyrosine kinase inhibitors, Biochem. Pharmacol. 60 (2000) 885-898. 
6. M. Barvian, R. J. Booth, J. Quin III., J. T. Repine, D. J. Sheehan, P. L. Toogood, S. N. Vanderwel and H. Zhou, 2-(Pyridin-2-yl-amino)-pyrido[2,3-d]pyrimidin-7-ones, PCT. Int. Appl. (2007), (C07D401/14; A61K31/444) WO 7208489.

7. H. B. Arzeno, J. J. Chen, J. P. Dunn, D. M. Goldstein and J. A. Lim, Preparation of 7-oxopyridopyrimidines as P38 MAP kinase inhibitors, PCT. Int. Appl. (2002), WO 200218379.

8. R. J. Booth, A. Chatterjee and T. C. Malone, Pyridopyrimidinone derivatives for treatment of neurodegenerative disease, PCT. Int. Appl. (2001), WO 2001055148.

9. S. Tu, J. Zhang, X. Zhu, J. Xu, Y. Zhang, Q. Wang, R. Jia, B. Jiang and J. Zhang, New potential inhibitors of cyclin-dependent kinase: Design and synthesis of pyrido[2,3-d]pyrimidine derivatives under microwave irradiation, Bioorg. Med. Chem. Lett. 16 (2006) 3578-3581; DOI: 10.1016/j. bmcl.2006.03.084.

10. M. Hájek, N. Matulová, I. Votruba, A. Holý and E. Tlouš'tova, Inhibition of human, telomerase by diphosphates of acyclic nucleoside phosphonates, Biochem. Pharmacol. 70 (2005) 894-900; DOI: 10.1016/j.bcp.2005.06.07.

11. J. J. Chen, J. P. Dunn, D. M. Goldstein and C. M. Stahl, Preparation of 2,6-disubstituted 7-oxopyrido[2,3- $d]$ pyrimidines for treating P38 mediated disorders, PCT. Int. Appl. (2002) WO 2002064594.

12. S. R. Kanth, G. V. Reddy, K. H. Kishore, P. S. Rao, B. Narsaiah and U. S. N. Murthy, Convenient synthesis of novel 4-substituted amino-5-trifluoromethyl-2,7-disubstituted pyrido[2,3- $d]$ pyrimidines and their antibacterial activity, Eur. J. Med. Chem. 41 (2006) 1011-1016; DOI: 10.1016/j.ejmech. 2006.03.028.

13. V. Darias, S. S. Abdallah, M. L. Tello, L. D. Delgado and S. Vega, NSAI activity study of 4-phenyl-2-thioxo-benzo[4,5]thieno[2,3-d]pyrimidines derivatives, Arch. Pharm. 327 (1994) 779-383; DOI: 10.1002/ardp. 19943271205.

14. L. Cordeu, E. Cubedo, E. Bandres, A. Rebollo, X. Saenz, H. M. Chozes, V. Dominquez, M. Echeverria, B. Mendivil, C. Sanmartin, J. A. Palop, M. Font and J. Garcia-Foncillas, Biological profile of new apoptotic agents based on 2,4-pyrido[2,3-d]-pyrimidine derivatives, Bioorg. Med. Chem. 15 (2007) 1659-1669; DOI: 10.1016/j.bmc.2006.12.010.

15. M. Zink, H. Lanig and R. Troschütz, Structural variations of piritrexim, a lipophilic inhibitor of human dihydrofolate reductase: synthesis, antitumor activity and molecular modeling investigations, Eur. J. Med. Chem. 39 (2004) 1079-1088; DOI: 10.1016/j.ejmech.2004.09.001.

16. R. A. Newberry and B. J. Bushell, Thiazole derivatives U.S. 3, 979, 402 (Cl.260-302R; Co7 D227122). 07 Sep. (1976), Brit. Appl. 748, 870, 27 Feb. (1974); ref. Chem. Abstr. 86 (1977) 29795z.

17. M. Yu. Gavrilov, L. G. Mardanova, V. E. Kolla and M. E. Konshin, Synthesis and anti-inflammatory and analgesic activities of 2-arylamino-5,6,7,8-tetrahydroquinoline-3-carboxamides, J. Pharm. Chem. 22 (1988) 554-556; DOI: 10.1007/BF00763528.

18. A. B. A. El-Gazzar, A. M. Gaafar, H. N. Hafez and A. M. Abdel-Fattah, Regioselective synthesis and reactions of polynuclear heterocyclic derived from pyrido[2,3- $d]$ pyrimidines, with new ring system, Phosphorus, Sulfur, Silicon 182 (2007) 369-403; DOI: 10.1080/10426500600919967.

19. Guide for the Care and Use of Animals (Eds. E. D. Olfert, B. M. Cross and A. A. McWilliam), $2^{\text {nd }}$ ed, Vol. I, Canadian Council on Animal Care, Ottawa 1993, p. 211.

20. C. A. Winter, E. A. Risley and G. W. Nuss, Carragenan-induced edema in hind paw of the rat as an assay for anti-inflammatory drugs, Proc. Soc. Exp. Biol. Med. 111 (1962) 544-547.

21. O. O. Adeyemi, O. S. Okpo and O. J. Okpaka. The analgesic effect of the methanolic extract of acanthus montanus, Ethnopharmacology 90 (2004) 45-48; DOI: 10.1016/j.jep.2003.09.021.

22. V. Cioli, S. Putzolu, V. Rossi, S. P. Barrcellona and C. Corradino, The role of direct tissue contact in the production of gastro-intestinal ulcers by anti-inflammatory drugs in rats, Toxicol. Appl. Pharmacol. 50 (1979) 283-289. 
H. N. Hafez et al.: Synthesis and evaluation of analgesic, anti-inflammatory and ulcerogenic activities of some triazolo- and 2-pyrazolyl-pyrido[2,3-d]-pyrimidines, Acta Pharm. 58 (2008) 359-378.

$S A \check{Z} E T A K$

\section{Sinteza i vrednovanje analgetskog, protuupalnog i ulcerogenog djelovanja nekih triazolo- i 2-pirazolil-pirido[2,3-d]-pirimidina}

HEND N. HAFEZ, HEBAT-ALLAH S. ABBAS i ABDEL-RAHMAN B. A. EL-GAZZAR

U radu je opisana sinteza serije 2-hidrazino-7,8-dihidro-6H-ciklopenta[5,6]pirido[2, 3-d]pirimidina i njihovih 1,7,8,9-tetrahidrociklopenta[5,6]pirido[2,3- $d][1,2,4]$ triazolo[4,3-a]pirimidinskih, 1,7,8,9-tetrahidrociklopenta[5,6]pirido[2,3-d][1,2,3,4]tetrazolo[4,5-a]pirimidinskih, 8,9-dihidro-7H-ciklopenta[5,6]pirido[2,3-d]imidazolo[1,2-a]pirimidinskih i 2-(pirazol-1-il)-7,8-dihidro-6H-ciklopenta[5,6]pirido[2,3-d]pirimidinskih derivata s potencijalnim protuupalnim i analgetskim te manjim ulcerogenim djelovanjem. Spojevima $\mathrm{s}$ izraženim protuupalnim djelovanjem testirano je analgetsko i ulcerogeno djelovanje. Spojevi 3-amino-6-(4-aril)-9-(4-arilmetilen)-ciklopenta[5,6]pirido[2,3- $d][1,2,4]$ triazolo[4,3-a ] pirimidin-5(H)-on (4c), 1-amino-2-metil-6-(4-aril)-9-(4-aril-metilen)-ciklopenta[5,6]pirido [2, 3- $d]$ imidazolo[1,2-a]pirimidin-5(H)-on (6a), 2-amino-5-(4-aril)-8-(4-arilmetilen)-ciklopenta[5,6]pirido[2,3- $d$ ] pirimidin-4(H)-on (9), 2-(3-amino-5-hidroksipirazol-1-il)-5-(4-aril)-8-(4-arilmetilen)-ciklopenta[5,6]-pirido[2,3-d]pirimidin-4(H)-on (10a) i 3-tiokso-6-(4-aril)-9-(4-arilmetilen)-ciklopenta[5,6]pirido[2,3- $d][1,2,4]$ triazolo[4,3-a]pirimidin-5(H)-on (13) pokazali su značajno analgetsko djelovanje. Spoj 2-(3-amino-5-hidroksipirazol-1-il)-5-(4-aril)-8-(4-arilmetilen)-ciklopenta[5,6]pirido[2,3-d]pirimidin-4(H)-on (10a) je vodeći spoj s jačim protuupalnim djelovanjem $(82,8 \%)$ od ibuprofena $(79,5 \%)$, a slabijim ulcerogenim djelovanjem.

Ključne riječi: pirido[2,3-d]pirimidini, [1,2,4]triazol anelacija, protuupalno djelovanje, analgetsko djelovanje

National Research Centre, Photochemistry Department (Heterocyclic Unit), Dokki, 12622 Cairo, Egypt 